\title{
Insetos indutores de galhas da porção sul da Cadeia do Espinhaço, Minas Gerais, Brasil
}

\author{
Marco Antonio A. Carneiroํㅡ, Rafael A. X. Borges ${ }^{2}$, Ana P. A. Araújo ${ }^{3}$ \& G. Wilson Fernandes ${ }^{4}$
}

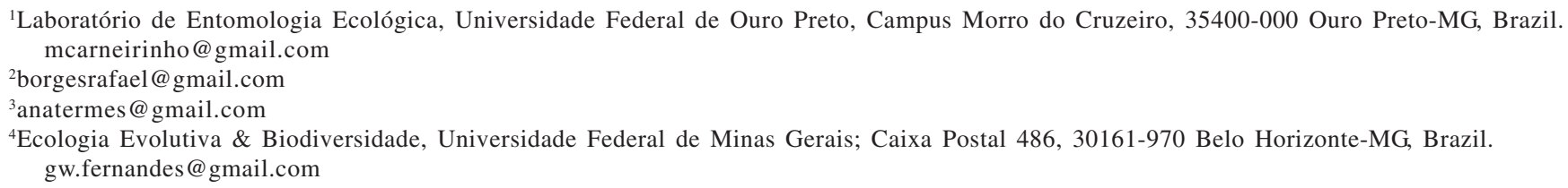

\begin{abstract}
Gall inducing insects from southern portion of the Espinhaço Range, Minas Gerais, Brazil. Galling species richness is higher at intermediate latitudes on warm habitats and sclerophyllous vegetation under water and nutrient stress. In the tropical region, galling species richness is higher in rupestrian fields. Here the gall-inducing insects and their host plants of the southern portion of the Espinhaço range, southeastern Brazil are described. 60 study sites from six distinct regions along the Espinhaço range, at Minas Gerais state were selected. In each study site 100 plants were sampled summing up to 6,000 plants censused along an altitudinal gradient ranging from $668 \mathrm{~m}$ to $1860 \mathrm{~m}$ a.s. 1 . A total of 241 distinct galls morphotypes were found on 142 host plant species belonging to 29 families from a total 384 species censused. The richest galling fauna was found in the Asteraceae (42\%), mainly the genus Baccharis. The largest proportion of galls $(85 \%)$ was induced by Cecidomyiidae followed by Lepidoptera (4\%) and Homoptera (3\%). Stems were the most frequently galled plant organ (72\%) while the most common shapes were elliptical (37\%) and globoid (36\%). The host plant species presenting the highest number of gall morphotypes types was Baccharis pseudomyriocephala with 10 galls. This study supported the assertion that rupestrian fields present the highest galling species richness.
\end{abstract}

KEYWORDS. Biodiversity; cerrado; host plant family; mountain; rupestrian fields.

RESUMO. Insetos indutores de galhas na porção sul da Cadeia do Espinhaço, Minas Gerais, Brasil. A riqueza de insetos galhadores é maior nas latitudes intermediárias em hábitats quentes e com vegetação esclerófila sob estresse hídrico e de nutrientes. Em regiões tropicais, os campos rupestres são indicados como hábitats ricos em espécies de insetos galhadores. Neste trabalho, foram descritas as galhas induzidas por insetos e suas plantas hospedeiras na porção sul da Cadeia do Espinhaço, sudeste do Brasil. Foram selecionados 60 sítios em seis regiões ao longo na porção sul da Cadeia do Espinhaço no estado de Minas Gerais. Em cada sítio 100 plantas foram amostradas totalizando 6.000 plantas censuradas ao longo de um gradiente altitudinal de 668 a 1860m. Foram encontrados 241 morfotipos de galhas em 142 espécies de plantas distribuídas em 29 famílias e de um total de 384 espécies de plantas amostradas. As famílias mais ricas em espécies de insetos galhadores foram Asteraceae (42\%), principalmente espécies do gênero Baccharis. A maior parte das galhas ( $85 \%)$ foi induzida por insetos da família Cecidomyiidae seguidos por Lepidoptera (4\%) e Homoptera (3\%). Os ramos foram os órgãos mais freqüentemente atacados (72\%) enquanto que os morfotipos mais comuns foram o elíptico (37\%) e o globóide (36\%). A espécie de planta hospedeira que apresentou mais morfotipos de galhas foi Baccharis pseudomyriocephala com 10 galhas distintas. Este estudo sustenta a afirmativa que campos rupestres apresentam uma elevada riqueza em espécies de insetos galhadores.

PALAVRAS-CHAVE. Biodiversidade; campos rupestres; cerrado; família de plantas hospedeiras; montanhas.

Galhas são alterações provocadas pela mudança do padrão de crescimento e desenvolvimento de tecidos ou órgãos da planta em resposta à ação de um inseto indutor (Dreger-Jauffret \& Shorthouse 1992). As galhas são caracterizadas pelo aumento do número e/ou tamanho das células culminando na formação de uma estrutura simétrica (Raman 2007). Os insetos galhadores representam os herbívoros mais sofisticados, capazes de controlar e redirecionar a planta hospedeira em seu benefício (Abrahamson \& Weis 1997; Shorthouse et al. 2005).

A galha é uma entidade fenótipica que representa a interação entre o genótipo do inseto, o genótipo da planta hospedeira e o meio ambiente (Weis et al. 1988). Do ponto de vista evolutivo, galhas podem ser vistas como fenótipos estendidos dos insetos ou suas adaptações para se alimentarem de tecidos de alta qualidade nutricional, proteção contra ataque de inimigos naturais e as variações do meio ambiente (Price et al. 1986; Weis et al. 1988; Nyman \& JulkunenTiitto 2000; Stone \& Schönrogge 2003). Insetos indutores de galhas são muito ricos em espécies e as últimas estimativas apontam para a existência de 21000 a 211000 espécies na Terra (Espírito-Santo \& Fernandes 2007). Na região Neotropical, particularmente as regiões de cerrados e campos rupestres são apontadas como as mais ricas em espécies de insetos galhadores do mundo (Lara \& Fernandes 1996).

O número de espécies de insetos indutores de galhas varia enormemente entre famílias e gêneros de plantas hospedeiras em diferentes regiões geográficas. No Brasil, algumas famílias de plantas são destacadamente ricas em insetos galhadores, entre elas Fabaceae, Asteraceae, Myrtaceae, Malpighiaceae, 
Rubiaceae e Bignoniaceae (Fernandes et al. 1997, 2001; Gonçalves-Alvim \& Fernandes 2001a, b; Julião et al. 2002; Urso-Guimarães et al. 2003; Urso-Guimarães \& Scarelli-Santos 2006; Mendonça 2007).

O cerrado brasileiro é considerado uma das formações vegetais mais ameaçadas na atualidade e foi incluído na lista de "hotspots" de diversidade do planeta (Myers et al. 2000; Jepson 2005). Este bioma ocupa $22 \%$ do território brasileiro ou uma área aproximada de 2,0 milhões de $\mathrm{km}^{2}$, sendo uma savana tropical com uma flora vascular das mais ricas em espécies (Oliveira \& Marquis 2002; Ratter et al. 2006; Silva et al. 2006). Apresenta diferentes tipos de fisionomias vegetais ao longo de uma grande variedade ambiental controlada pelo tipo de solo, clima e topografia (Oliveira \& Marquis 2002; Silva et al. 2006). Restritos ao Bioma Cerrado, a fitofisionomia de campos rupestres ocupa o topo dos platôs e das cadeias montanhosas localizados nos estados de Minas Gerais, Bahia e Goiás (Giulietti et al. 1987; Giulietti \& Pirani 1988; Safford 1999; Oliveira-Filho \& Ratter 2002). O número de espécies endêmicas da flora e da fauna nos campos rupestres associados à Cadeia do Espinhaço é muito elevado, e de acordo com os dados publicados até o momento, de $17 \%$ a $31 \%$ das espécies são endêmicas (Giulietti et al. 1987; Giulietti \& Pirani 1988; Safford 1999; Rapini et al. 2002). Além de disto os ambientes montanhosos do sudeste brasileiro são responsáveis pelo abastecimento de água de $25 \%$ da população brasileira (Safford 1999).

Este trabalho faz parte de um projeto para descrever a história natural dos insetos galhadores, suas galhas e plantas hospedeiras de campos rupestres. Para isso as galhas foram descritas e caracterizadas através de sua morfologia externa e ocorrência nas plantas hospedeiras (Fernandes \& Price 1988; Fernandes et al. 1996).

\section{MATERIALE MÉTODOS}

As amostragens foram concentradas em regiões de campos rupestres no estado de Minas Gerais ao longo da Cadeia do Espinhaço. A Cadeia do Espinhaço compreende um grupo de serras que se estende por cerca de $1.100 \mathrm{~km}$ na direção N-S e 50 a $100 \mathrm{~km}$ na direção E-W, entre os limites $20^{\circ} 35^{\prime} \mathrm{S}$ e $11^{\circ} 11^{\prime} \mathrm{S}$, indo da Serra de Ouro Branco, em Minas Gerais (MG), até a Serra da Jacobina, na Bahia (BA) (Derby 1906; Giulietti et al. 1987; Pirani et al. 2003). Formada por soerguimentos intermitentes a partir do Paleozóico, a Cadeia do Espinhaço é formada por dois principais setores, o planalto de Diamantina (MG) e a Chapada Diamantina (BA), separados por uma grande extensão de terras baixas onde pequenas serras isoladas ficam reduzidas e afastadas (Pirani et al. 2003). As serras da Cadeia do Espinhaço possuem altitudes médias entre 800 e $2000 \mathrm{~m}$ (Moreira 1977). A Cadeia do Espinhaço apresenta solos rasos, arenosos e pobres em nutrientes (Benites et al. 2007); um clima do tipo Cwb de Köppen (clima mesotérmico com verões brandos e estação chuvosa no verão), com temperaturas médias entre $17,4^{\circ}$ e $19,8^{\circ} \mathrm{C}$, sendo a temperatura média do mês mais quente inferior a $22^{\circ} \mathrm{C}$ (Galvão \& Nimer 1965). A precipitação anual da região está em torno de $1.500 \mathrm{~mm}$, com um inverno seco de 3 a 4 meses, e um período úmido de 7 a 8 meses.

Devido às facilidades logísticas fornecidas pelas Unidades de Conservação instaladas nestas regiões, foram selecionadas ao longo da Cadeia do Espinhaço seis regiões (P. E. do Itacolomi, Serra de Ouro Branco, R. P. P. N. Serra do Caraça, P. E. do Rio Preto, P. E. do Grão Mogol, e P. E. do Biribiri). Foram amostradas 10 áreas em cada região totalizando sessenta pontos de coleta, ao longo de um gradiente altitudinal de $1.172 \mathrm{~m}$ de extensão ( 688 a $1.860 \mathrm{~m}$ ). O gradiente altitudinal no P. E. do Itacolomi foi de 1.229 a $1.684 \mathrm{~m}$; na Serra de Ouro Branco, de 1.087 a 1.579 m; na R. P. P. N. Serra do Caraça, de 1.094 a $1.860 \mathrm{~m}$, no P. E. do Rio Preto, de 773 a 1.395 m, no P. E. do Grão Mogol, de 688 a 1.230 m e no P. E. do Biribiri, de 724 a $1.481 \mathrm{~m}$.

As amostras nas altitudes mais elevadas foram feitas em áreas de afloramento rochoso e campo rupestre arbustivo. Nas altitudes intermediárias e baixas, foram amostrados em áreas ecotonais de cerrado/campo rupestre ou em campo cerrado. As áreas foram selecionadas arbitrariamente e sempre que possível foram selecionados locais preservados, sem sinal de pisoteio de gado ou fogo recente e longe de trilhas.

A riqueza em espécies de insetos indutores de galhas pode variar entre diferentes tipos arquitetônicos de plantas sendo os arbustos o tipo arquitetônico que apresenta o maior número de espécies de insetos galhadores (Fernandes et al. 1996; Price et al. 1997). Para padronizar as coletas, as galhas foram amostradas somente em espécies de plantas lenhosas de porte arbustivo entre 0,3 a 2,0m de altura. Além disso, o censo de galhas fica facilitado, uma vez que, é realizado de maneira direta sobre a copa das plantas (Fernandes \& Price 1988).

A amostragem de galhas foi realizada seguindo a metodologia descrita por Fernandes \& Price (1988, mas veja também Price et al. 1998). Em todas as regiões cada área amostrada correspondeu a uma parcela com 100 plantas lenhosas em cada ponto de amostra, totalizando 1.000 plantas em cada região e 6.000 plantas em todo o trabalho. Em cada planta foi amostrada toda a parte aérea do indivíduo através da contagem direta do número de morfotipos de galhas.

Insetos indutores de galhas são poucos conhecidos, e a maioria das suas espécies é nova para a Ciência (Gagné 1994). As morfoespécies de galhas foram identificadas com base na sua morfologia externa em combinação com o órgão e a espécie da planta hospedeira. De maneira geral, cada espécie de inseto induz uma galha de morfologia típica em um único órgão de uma determinada espécie de planta hospedeira (Dreger-Jauffret $\&$ Shorthouse 1992; Floate et al. 1996). No Brasil, por exemplo, 95\% das espécies de Cecidomí́deos conhecidas podem ser discriminadas com base no morfotipo de galha associado à planta hospedeira (Carneiro et al. 2009). Por isso, a separação em morfotipos pode ser utilizada para representar a riqueza em espécies de IIG (Price et al. 1998; Cuevas-Reyes et al. 2004; Carneiro et al. 2009). As galhas foram descritas e categorizadas de acordo com as suas características morfológicas externas como, forma, presença de pêlos (a não ser os ocorrentes "naturalmente" na planta hospedeira), se ocorre em 
agrupamentos ou isoladas, e órgão atacado do hospedeiro (Fernandes et al. 1996, 1997, 2001; Stone \& Schönrogge 2003).

As amostras das plantas hospedeiras e suas galhas foram herborizadas e depositadas nos herbários OUPR e BHCB (acrônimos segundo Holmgren et al. 1990). As plantas coletadas foram separadas em famílias e sempre que possível identificada até a categoria taxonômica de espécie. Dos 6.000 indivíduos e 384 espécies de plantas coletadas, 5.322 (89\%) indivíduos e 252 (66\%) espécies de plantas foram identificados até o nível de espécie. A classificação das espécies de plantas hospedeiras seguiu o sistema proposto pelo Angiosperm Phylogeny Group II (APG II 2003).

\section{RESULTADOS}

Foram encontrados 241 tipos morfologicamente distintos de galhas de insetos e um total de 384 espécies de plantas. As galhas foram encontradas apenas em 142 espécies de plantas hospedeiras de 29 famílias e 78 gêneros (Tabela I). A riqueza de insetos galhadores foi maior no P. E. Rio Preto (75 morfotipos, $31 \%$ do total) seguidas pelo R. P. P. N. Caraça (71, 29\%), P. E. Biribiri (63, 26\%), P. E. Itacolomi (59, 24\%), Serra do Ouro Branco $(50,21 \%)$ e P. E. Grão $\operatorname{Mogol}(18,7 \%)$. Entre as regiões estudadas a maioria das galhas foi induzida por insetos da família Cecidomyiidae $(85 \%)$ seguido por Lepidoptera $(4 \%)$ e Homoptera (3\%). Foram encontradas duas galhas induzidas por nematóides em espécies de Miconia (Melastomataceae). As famílias que apresentaram um maior número de espécies de galhas foram: Asteraceae (42\%), Melastomataceae (11\%), Malpighiaceae (9\%) e Fabaceae (7\%). Os gêneros que apresentaram um maior número de espécies de galhas foram: Baccharis (16\%), Byrsonima (7\%), Microlicia, Lessingianthus e Lychnophora (4\% cada). A maior parte das galhas foi induzida no caule e nas gemas apicais $(72 \%)$ e as formas de galhas mais freqüentes foram a fusiforme $(37 \%)$ e a globosa (33\%). Noventa e quatro por cento das galhas não tinham pêlos e $85 \%$ ocorreram isoladamente sem a formação de aglomerados.

\section{DISCUSSÃO}

A maior parte dos trabalhos realizados no Brasil com descrição de galhas concentra-se no domínio do Cerrado (e.g., Gonçalves-Alvim \& Fernandes 2001b; Urso-Guimarães et al. 2003; Urso-Guimarães \& Scarelli-Santos 2006) e na vegetação de restinga (veja referências citadas em Maia 2005). Poucos foram os trabalhos realizados em outros biomas considerando a grande extensão territorial do Brasil, e.g. Mata Atlântica (Fernandes et al. 2001; Mendonça 2007), Pantanal (Julião et al. 2002), Floresta Amazônica (Julião et al. 2005) e mata sazonal seca (Coelho et al. 2009). Em áreas de campos rupestres, a descrição dos morfotipos de galhas e suas plantas hospedeiras estão disponíveis apenas para a Serra de São José (Maia \& Fernandes 2004) e para a região do município de Delfinópolis (Urso-Guimarães et al. 2003). Este trabalho relatou 241 morfotipos de galhas em 140 espécies de plantas (47 gêneros e 29 famílias) representando o maior levantamento de galhas feito em campos rupestres na Cadeia do Espinhaço.
Com base nas conclusões dos levantamentos acima citados de insetos galhadores e suas plantas hospedeiras algumas generalizações podem ser feitas. As famílias de plantas mais atacadas correspondem às mais representativas nas amostragens de galhadores em cada trabalho. Contudo, estas famílias também são as mais representativas nos biomas estudados (Giulietti et al. 1987; Giulietti \& Pirani 1988; Safford 1999; Hind 2003; Zappi et al. 2003; Almeida et al. 2004; Hatschbach et al. 2006). Por exemplo, o táxon com maior número de espécies (Asteraceae - 95 espécies) também apresentou o maior número de morfotipos de galhas (102 morfotipos). O mesmo padrão foi reconhecido no cerrado (Gonçalves-Alvim \& Fernandes 2001a, b), campos rupestres (Maia \& Fernandes 2004), floresta atlântica (Fernandes et al. 2001) e floresta sazonal subtropical (Mendonça 2007), onde famílias botânicas maiores, com maior número de espécies sustentam o maior número de espécies de insetos galhadores. Assim existe uma relação positiva entre número de insetos indutores de galhas e o tamanho do táxon da planta hospedeira, geralmente famílias ou gêneros (Fernandes 1992; Mendonça 2007).

Independentemente do tamanho do táxon, alguns táxons de plantas hospedeiras reconhecidamente têm uma baixa riqueza em espécies de insetos galhadores mesmo que intensamente amostrados, enquanto outros táxons têm uma grande riqueza em espécies mesmo que pouco amostrados (Hawkins \& Compton 1992). Insetos galhadores são extremamente diversos em determinados gêneros de plantas hospedeiras em diferentes regiões biogeográficas: cecidomiídeos em Baccharis (Neotropical, Gagné 1994; Fernandes et al. 1996) e Solidago (Neártica, Gagné 1994); cinipídeos em Quercus (Neártica e Paleártica, Ronquist 1994; Stone et al. 2002).

Os resultados obtidos neste estudo confirmam resultados prévios e de revisão da literatura e apontam Asteraceae como a família de planta hospedeira com maior número de espécies de insetos galhadores nos campos rupestres, principalmente as espécies do gênero Baccharis (Fernandes et al. 1996). Estes resultados refletem a grande riqueza em espécies de plantas deste gênero encontrado em áreas de campo rupestre (Barroso 1976; Munhoz \& Proença 1998; Hind 2003; Zappi et al. 2003; Hatschbach et al. 2006). Segundo Safford (1999), aproximadamente $5 \%$ de todas as espécies de plantas lenhosas em campos de altitude são do gênero Baccharis. Além disso, a grande maioria das galhas foi induzida por insetos da família Cecidomyiidae corroborando as afirmações de que estes insetos são os mais ricos em espécies na região neotropical (Gagné 1994; Maia 2005; Espírito-Santo \& Fernandes 2007).

A visão tradicional é que a maior parte das galhas é induzida nas folhas em comparação a outros órgãos (e.g. Dreger-Jauffret \& Shorthouse 1992). Resultados prévios em vegetação de campos rupestres mostram a mesma tendência, com folhas hospedando mais espécies de galhas (Maia \& Fernandes 2004). Neste estudo, a grande maioria das galhas descritas foi induzida em ramos e gemas. Na savana africana também foram encontradas mais galhas em ramos (Veldtman \& McGeoch 2003). Os autores sugerem que ramos são sítios mais seguros 

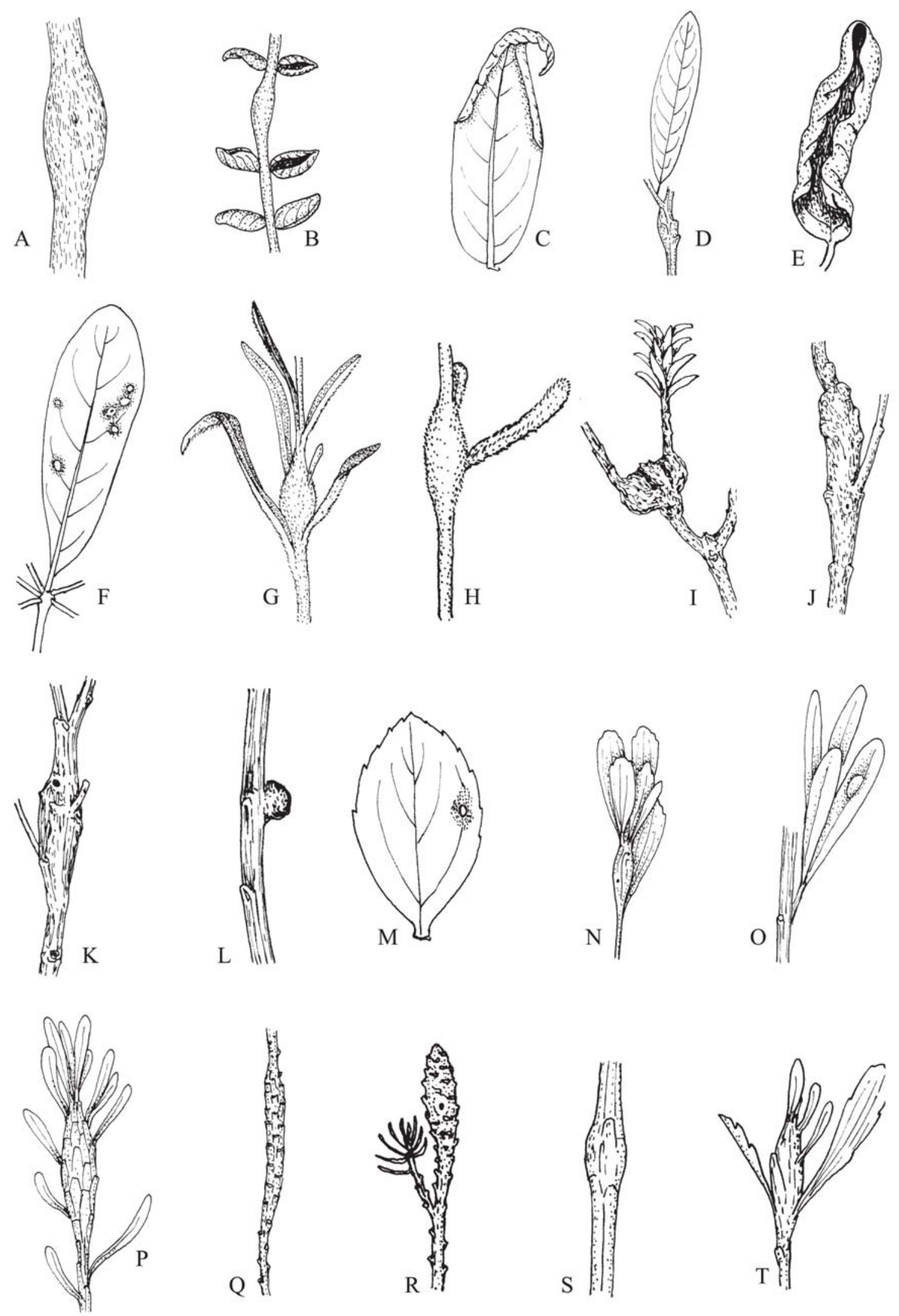

Fig. 1. Morfotipos de galhas em campos rupestres na porção sul da Cadeia do Espinhaço Para identificação das plantas hospedeiras veja a Tabela I. Anacardiaceae: Schinus terebinthifolius (A), Apocynaceae: Ditassa decussata (B); Himatanthus obovatus (C); Aquifoliaceae: Ilex aniara (D), Ilex brasiliensis (E) Araliaceae: Schefflera macrocarpus (F); Asteraceae: Achyrocline albicans (G), A. satureoides (H), Agrianthus microcilicoides (I), Aspilia fruticosa (J-L), Baccharis elliptica (M-N), B. leptocephala (O-P), B. minutiflora (Q-R), B. platypoda (S-T). 

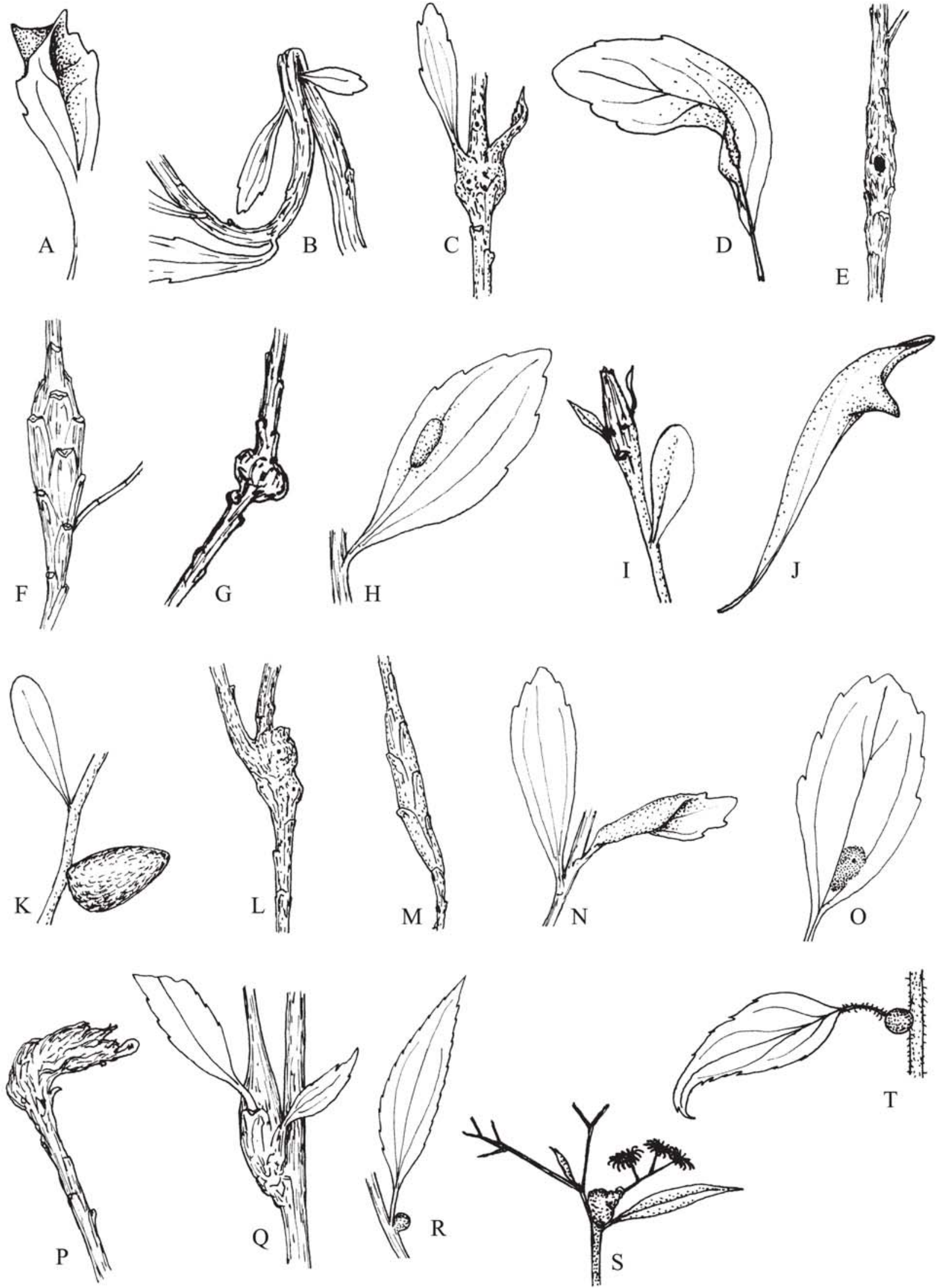

Fig. 2. Morfotipos de galhas em campos rupestres na porção sul da Cadeia do Espinhaço Para identificação das plantas hospedeiras veja a Tabela I. Asteraceae: Baccharis platypoda (A-D), B. reticularia (E-K), B. salzmanii (L-P), B. serrulata (Q-T). 

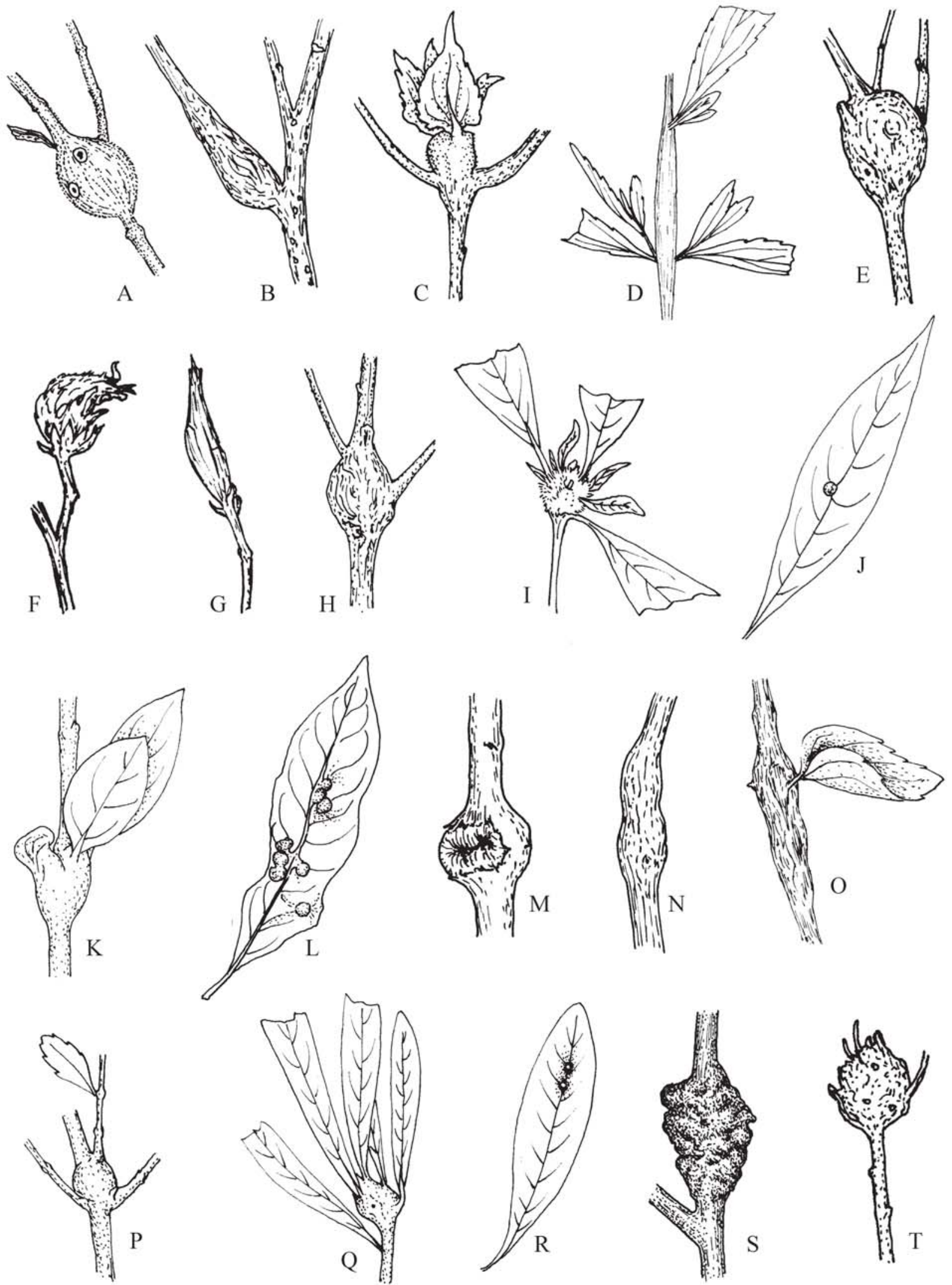

Fig. 3. Morfotipos de galhas em campos rupestres na porção sul da Cadeia do Espinhaço Para identificação das plantas hospedeiras veja a Tabela I. Asteraceae: Choromalaena horminoides (A), C. multiflosculosum (B-D), Choromalaena sp. (E), Dasyphylum fodinarum (F), Eremanthus erythropappus (G-I), Gochnatia sp1 (J), Hololepis pedunculata (K-M), Koanophyllon hebecladum (N), Lepidaploa lilacina (O-P), Lessingianthus elegans (Q), Lessingianthus linearifolius (R), Lessingianthus pychnostachius (S-T). 

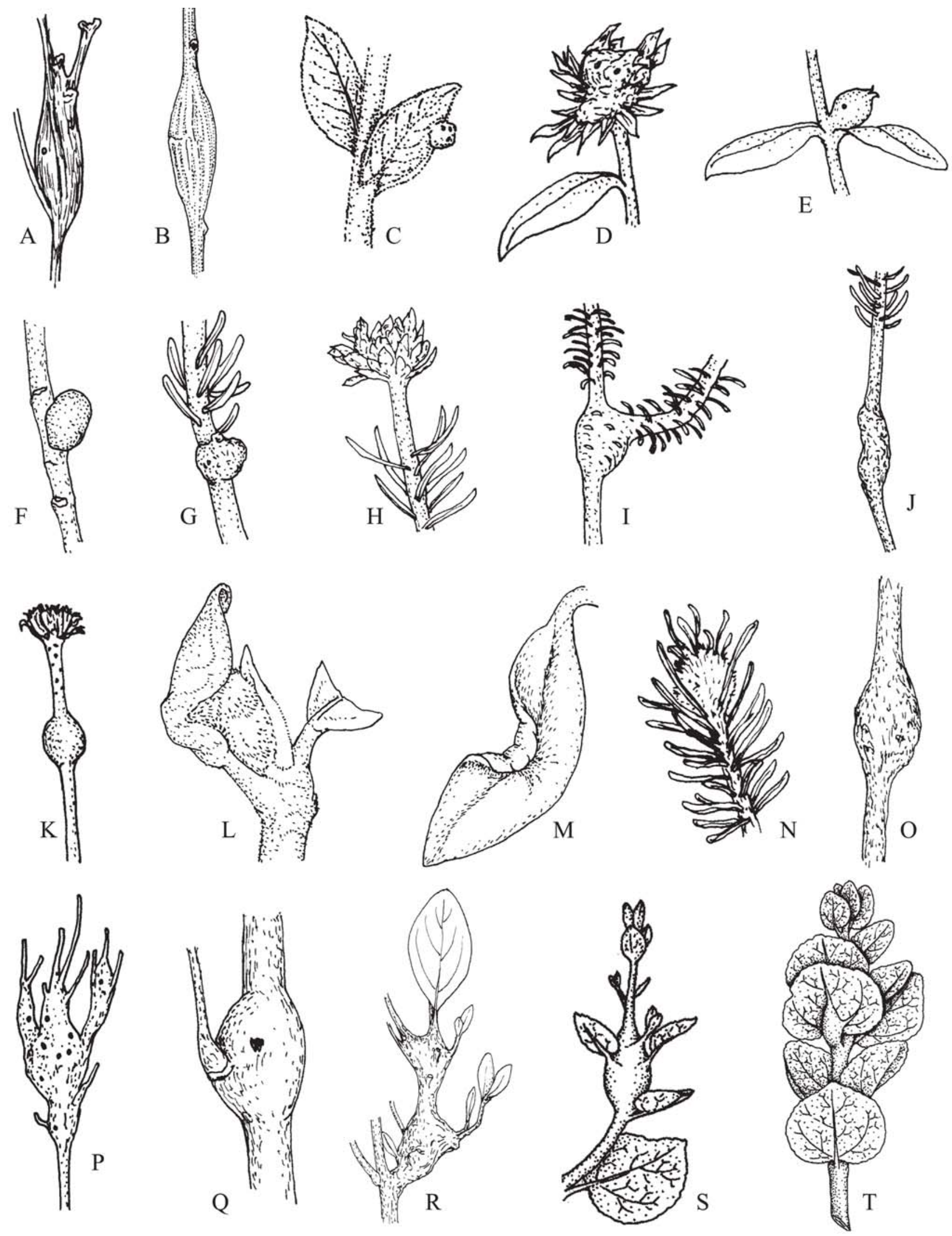

Fig. 4. Morfotipos de galhas em campos rupestres na porção sul da Cadeia do Espinhaço Para identificação das plantas hospedeiras veja a Tabela I. Asteraceae: Lessingianthus tomentellus (A-D), Lessingianthus warmingiana (E), Lourteigia ballotifolia (F), Lychnophora ericoides (G-H), L. passerina (I), L. ramosissima (J-K), L. tomentosa (L-M), L. trichocarpha (N-O), Mikania glabra (P-Q), M. glauca (R), M. nummularia (S-T). 

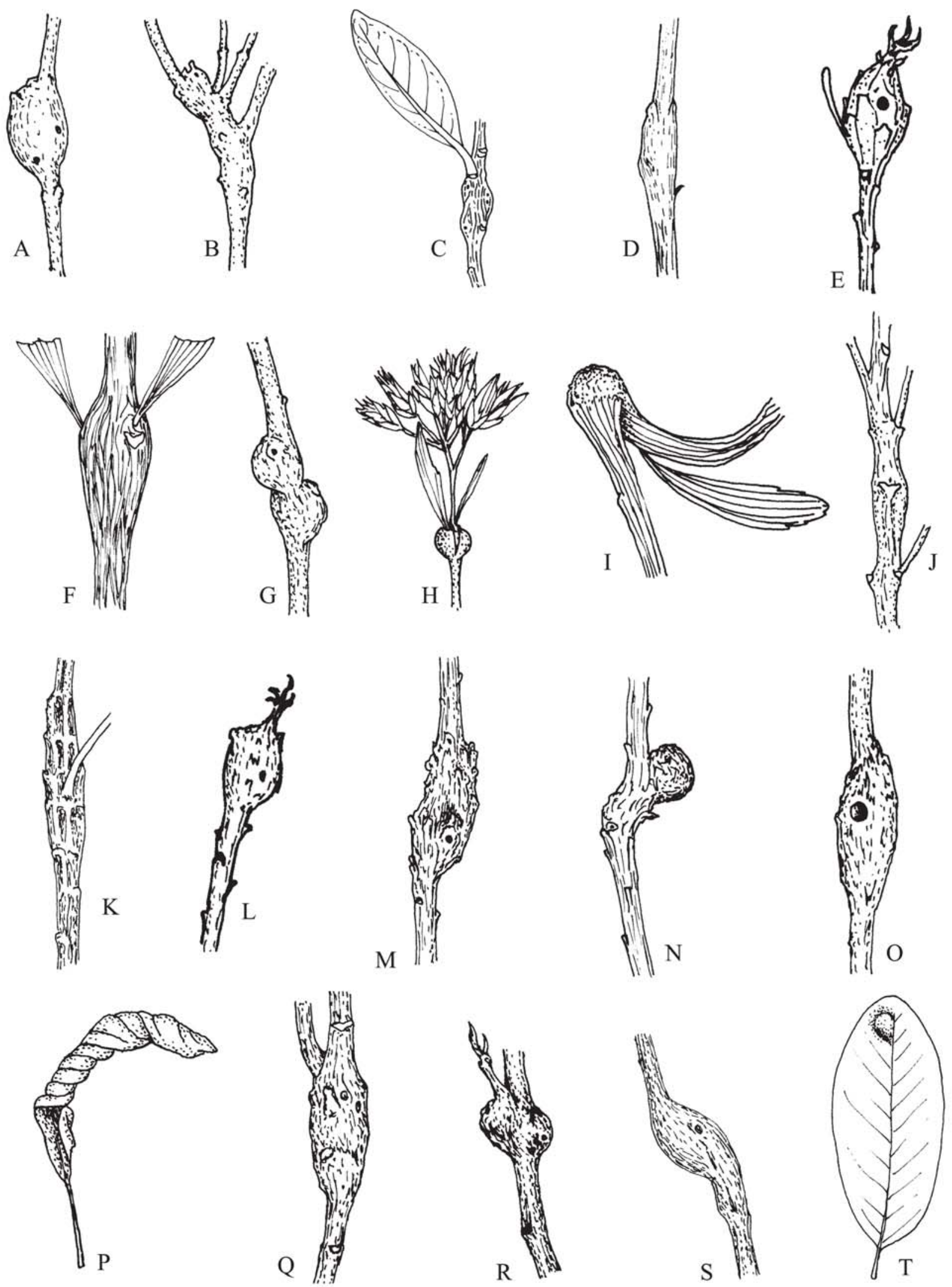

Fig. 5. Morfotipos de galhas em campos rupestres na porção sul da Cadeia do Espinhaço. Para identificação das plantas hospedeiras veja a Tabela I. Asteraceae: Mikania nummularia (A), M. parvifolia (B), Piptocarpha cf. axillaris (C), Piptolepis ericoides (D), Pseudobrickellia angustissima (E), Symphyopappus brasiliensis (F-I), S. reticulatus (J), Trichogonia hirtiflora (K), T. villosa (L-N), Bignoniaceae: Jacaranda paucifoliolata (OP), Tabebuia pumila (Q), Tabebuia sp.1 (R-S), Boraginaceae: Cordia verbenacea (T). 

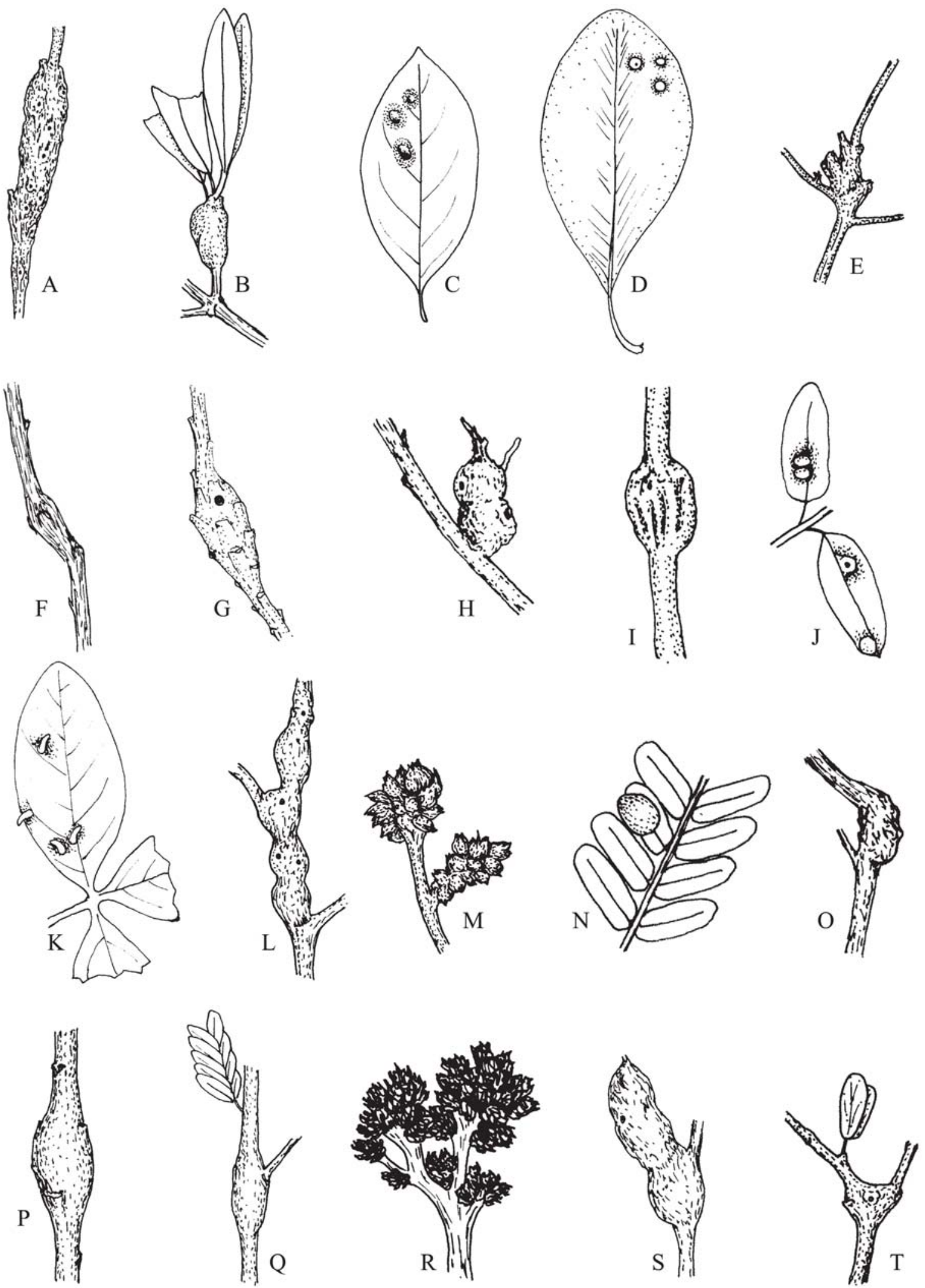

Fig. 6. Morfotipos de galhas em campos rupestres na porção sul da Cadeia do Espinhaço. Para identificação das plantas hospedeiras veja a Tabela I. Chrysobalanaceae: Coupeia sp. (A), Hirtella floribunda (B-C), Clusiaceae: Kielmeyera coriaceae (D), Connaraceae: Connarus suberosus (E), Convolvulaceae: Jacquemontia sp. (F), Ericaceae: Gaylussacia pinifolia (G-H), Euphorbiaceae: Croton staechadis (I), C. buxifolius (J), Manihot tripartita (K), Sebastiania aff. Marginata (L) Fabaceae: Bauhinia sp. 1 (M), Calliandra asplenioides (N), Chamaecrista catharticoides (O), C. chrsysocephala (P), C. cinerascens (Q), C. cf. cotinifolia (R), C. filicifolia (S), C. ramosa (T). 

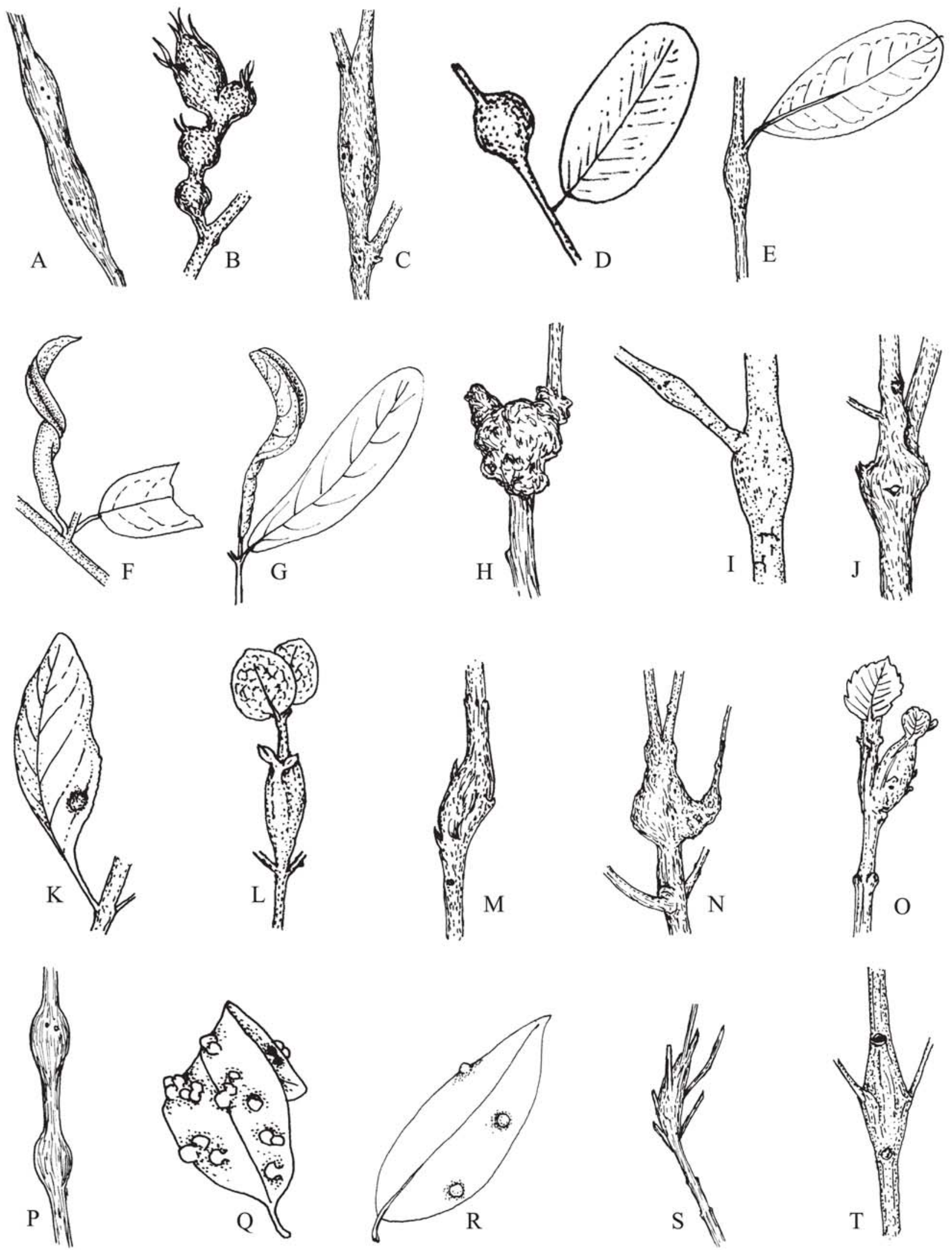

Fig. 7. Morfotipos de galhas em campos rupestres na porção sul da Cadeia do Espinhaço. Para identificação das plantas hospedeiras veja a Tabela I. Fabaceae: Chamaecrista repens (A-B), Dalbergia miscolobium (C-D), Periandra mediterranea (E-F), Senna sp. (G), Lamiaceae: Aegephila klotsckiana $(\mathrm{H})$, Eriope hypoleuca (I), Hyptis carpinifolia $(\mathrm{K})$, H. lacunosa $(\mathrm{L})$, H. lippioides $(\mathrm{M})$, H. sp1 (N-O), Lauraceae: Ocotea coymbosa (P), O. glauciana (Q), Lythraceae: Cuphea ericoides (R), Diplusodon buxifolius (S). 

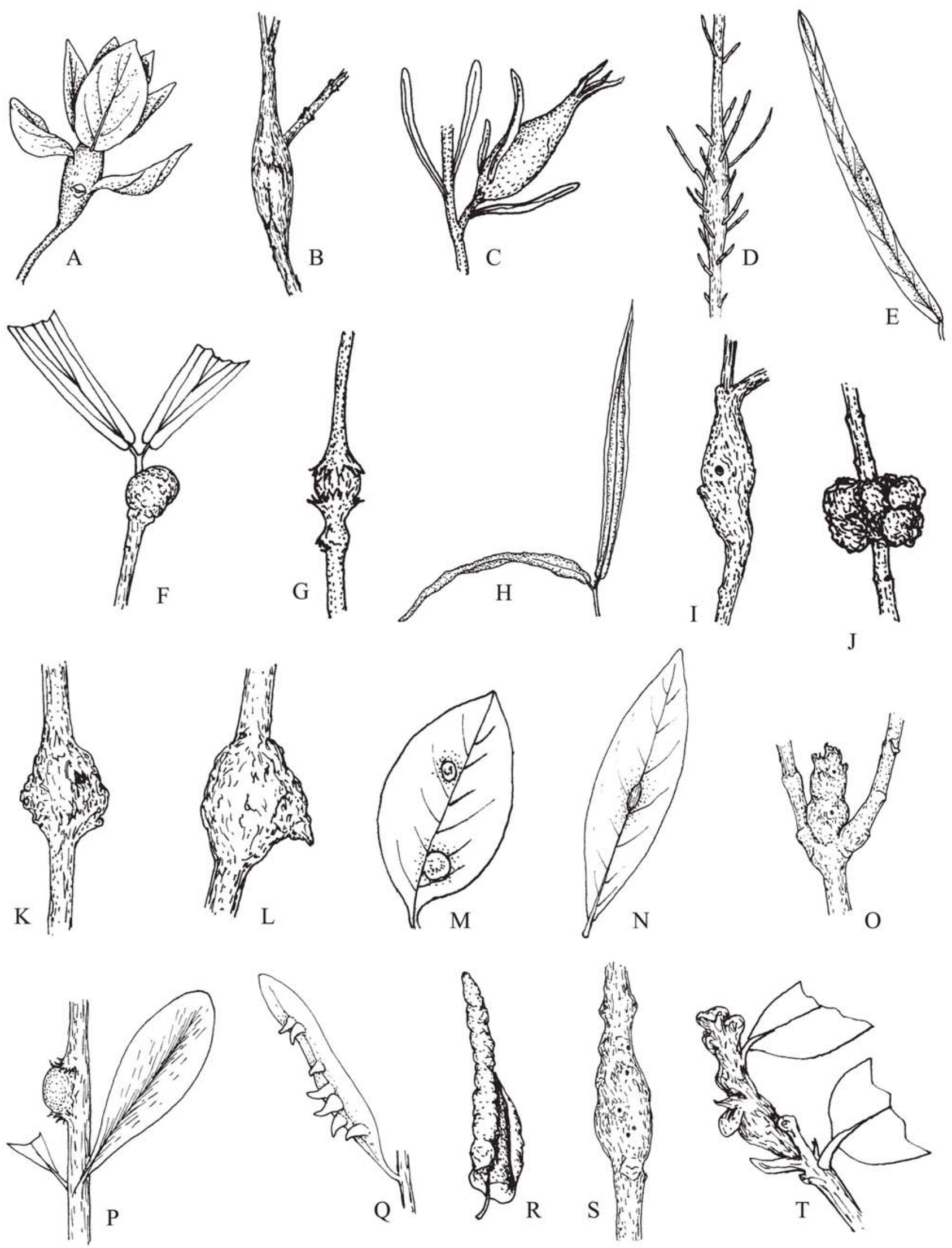

Fig. 8. Morfotipos de galhas em campos rupestres na porção sul da Cadeia do Espinhaço. Para identificação das plantas hospedeiras veja a Tabela I. Lythraceae: Diplusodon buxifolius (A), D. helianthenifolius (B), D. hexander (C-D), Malpighiaceae: Banisteriopsis angustifolia (E-H), B. malifolia (I), Byrsonima arctostaphylloides $(\mathrm{J})$, B. clausseniana $(\mathrm{K})$, B. coccolobifolia $(\mathrm{L}-\mathrm{R})$, B. variabilis $(\mathrm{S}-\mathrm{T})$. 

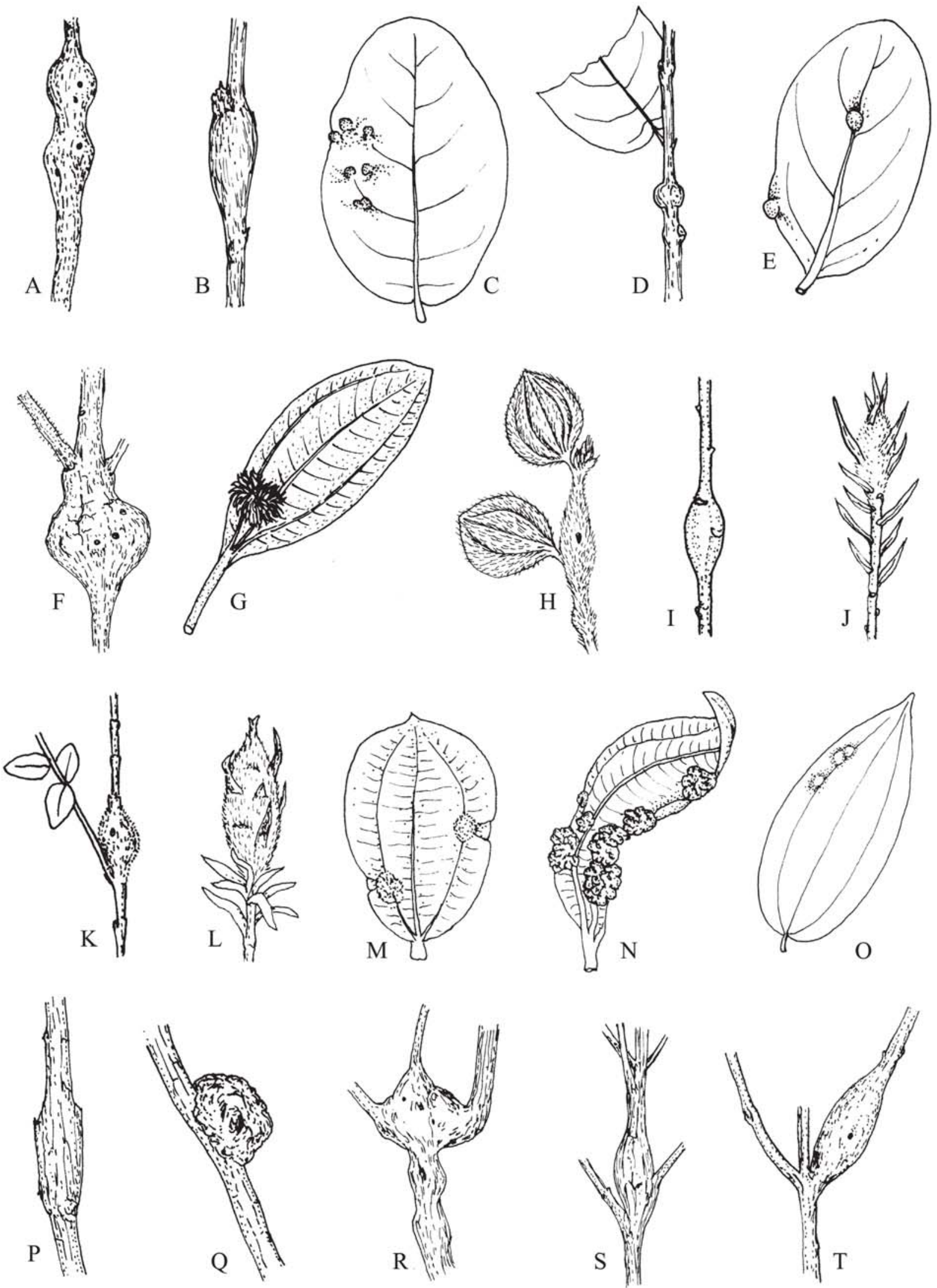

Fig. 9. Morfotipos de galhas em campos rupestres na porção sul da Cadeia do Espinhaço. Para identificação das plantas hospedeiras veja a Tabela I. Malpighiaceae: B. variabilis (A). Byrsonima sp. 1 (B-D), Peixotoa cipoana (E), Melastomataceae: Leandra aurea (F), Macairea radula (GH), Marcetia taxifolia (I-K), M. Hirsuta (L), Miconia albicans (M), M. coralina (N), Miconia sp. 1 (O-Q), Miconia sp. 2 (R), Microlicia cardiophora (S), M. confertiflora (T). 

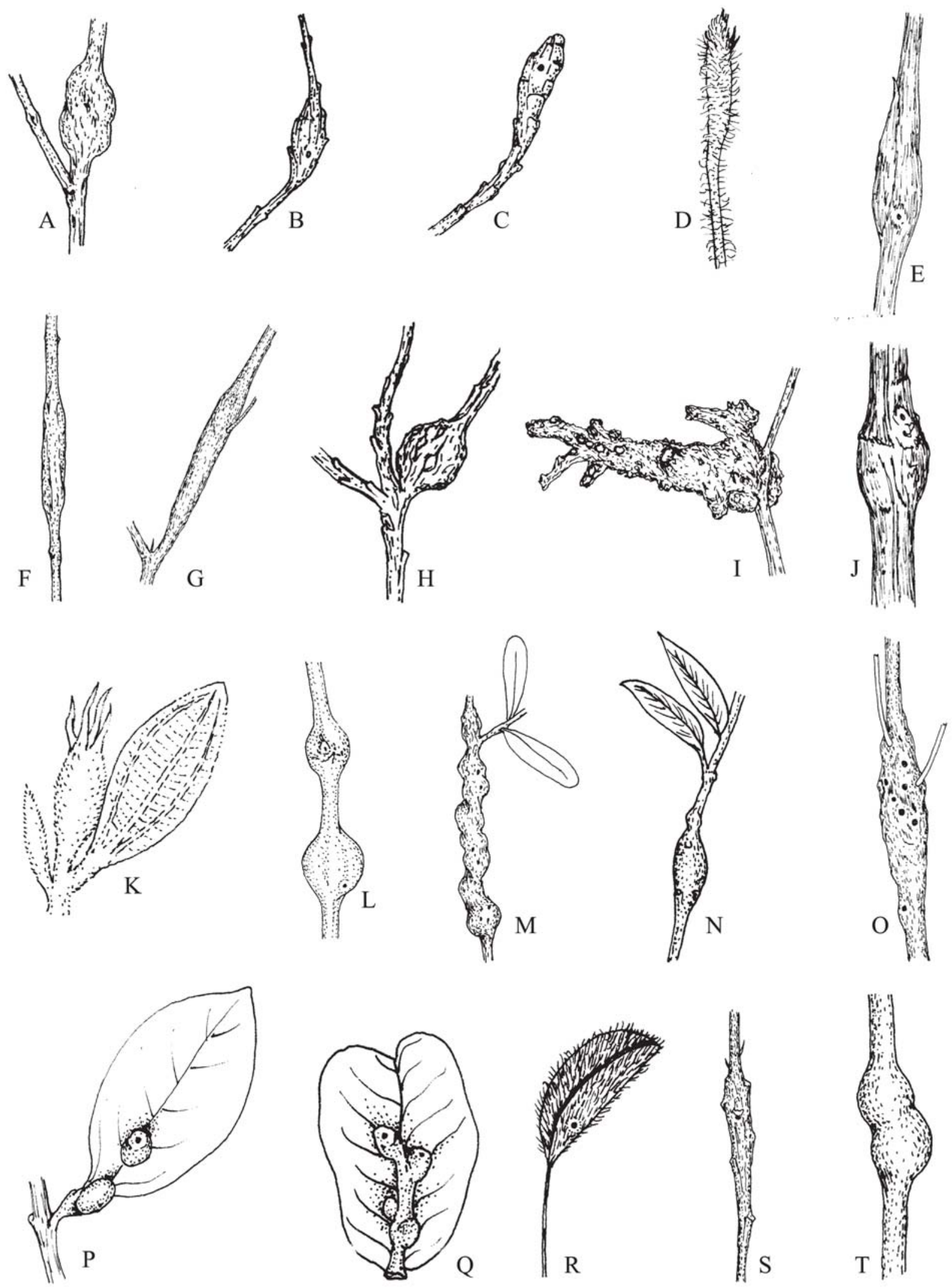

Fig. 10. Morfotipos de galhas em campos rupestres na porção sul da Cadeia do Espinhaço. Para identificação das plantas hospedeiras veja a Tabela I. Melastomataceae: Microlicia confertiflora (A), M. euphorbioides (B), M. fasciculata (C), M. graveolens (D), M. multicaules (E), M. suborticularifolia (F), Microlicia sp1 (G), Microlicia sp. $2(\mathrm{H})$, Tibouchina arenaria (I), T. multiflora (J), Trembleya laniflora (K), T. parviflora (L), Myrtaceae: Eugenia punicifolia (M), Myrcia montana (N), M. rufipes (O), Nyctaginaceae: Guapira graciliflora (P), G. roxia (Q), Oxalidaceae: Oxalis hirsutissima (R-S), Polygonaceae: Coccoloba acrostichoides (T). 

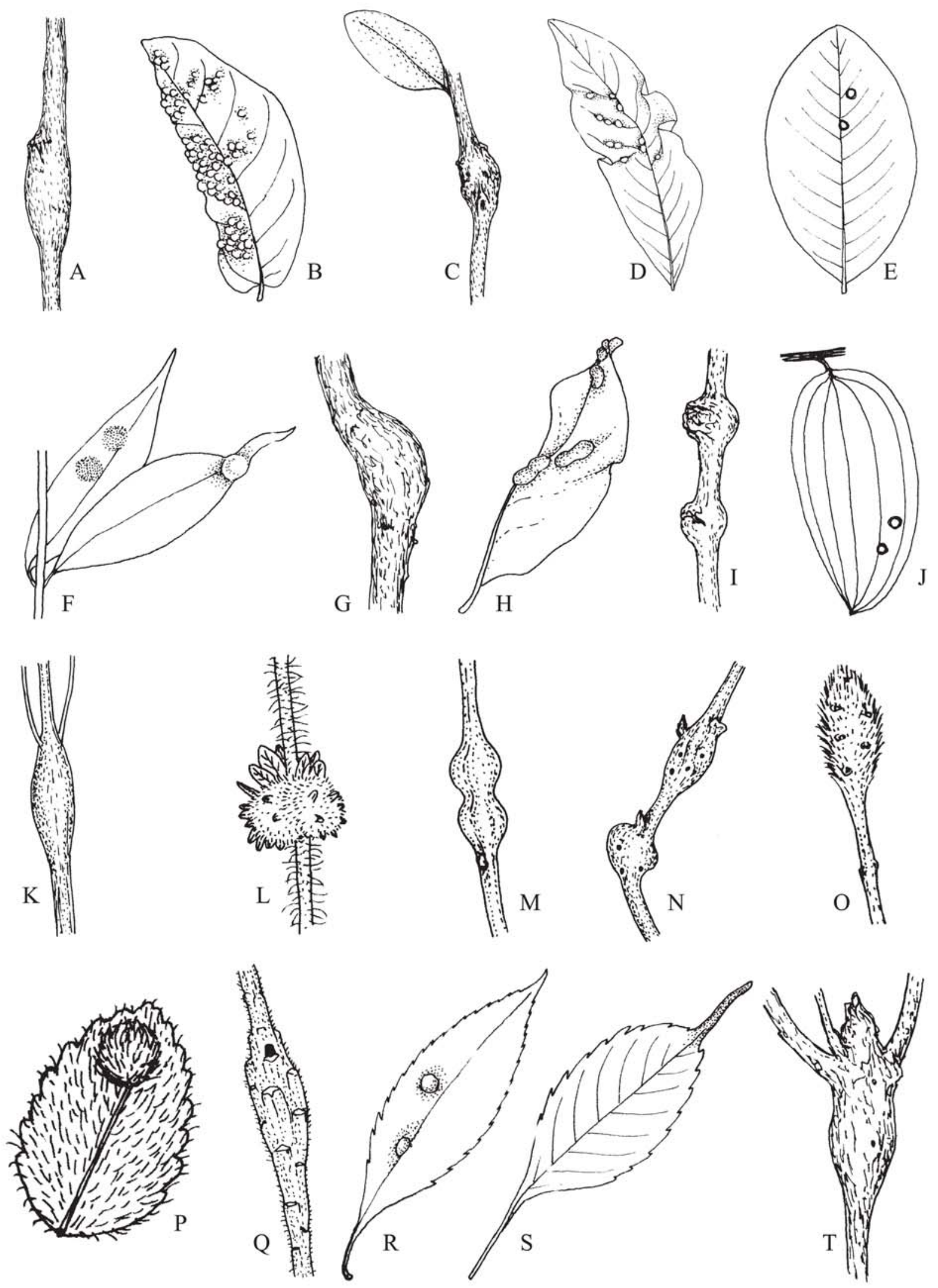

Fig. 11. Morfotipos de galhas em campos rupestres na porção sul da Cadeia do Espinhaço. Para identificação das plantas hospedeiras veja a Tabela I. Polygonaceae: Coccoloba acrostichoides (A-B); Rubiaceae: Cordiera concolor (C), Palicourea rigida (D-E), Psychotria vellosiana (F), Remijia ferruginea (G); Santalaceae: Phoradendron crassips (H); Sapindaceae: Matayba marginata (I); Smilacaceae: Smilax brasiliensis (J); Verbenaceae: Lantana lilacina (K-M), Lippia pohliana (N-P), Lippia pseudo-thea (Q), Stachytarpheta glabra (R-S), S. martiana (T). 
Tabela I. Plantas hospedeiras, descrição e local de ocorrência das galhas em campos rupestres em seis regiões na Cadeia do Espinhaço, Minas Gerais. $(\mathrm{PEI}=$ Parque Estadual $(\mathrm{P}$. E.) do Itacolomi, $\mathrm{SOB}=$ Serra do Ouro Branco, $\mathrm{SCA}=\mathrm{RPPN}$ do Caraça; PERP = P. E. Rio Preto; PEGM = P. E. Grão Mogol; PEBB = P. E. Biribiri).

\begin{tabular}{|c|c|c|c|c|c|}
\hline Planta hospedeira & Táxon galhador PEI SOB & SCA PERP PEGM & PEBB Órgão & Forma & Referên \\
\hline \multicolumn{6}{|l|}{ Anacardiaceae } \\
\hline Schinus terebinthifolius Raddi & Cecidomyiidae & $\mathrm{X}$ & caule & elíptica & Fig. 1A \\
\hline \multicolumn{6}{|l|}{ Apocynaceae } \\
\hline $\begin{array}{l}\text { Ditassa decussata Mart. } \\
\text { Himatanthus obovatus (Mi }\end{array}$ & $\begin{array}{l}\text { Cecidomyiidae } \\
\text { Homoptera }\end{array}$ & $\mathrm{X}$ & $\begin{array}{l}\text { caule } \\
\text { folha }\end{array}$ & $\begin{array}{l}\text { elíptica } \\
\text { borda enrolada }\end{array}$ & $\begin{array}{l}\text { Fig. 1B } \\
\text { Fig. 1C }\end{array}$ \\
\hline
\end{tabular}

Arg.) Woodson

\section{Aquifoliaceae}

Ilex amara (Vell.) Loes. Cecidomyiidae X Ilex brasiliensis Loes.

Homoptera

\section{Araliaceae}

Schefflera macrocarpa (Cham. \& Cecidomyiidae

Schltdl.) Frodin

\section{Asteraceae}

Achyrocline albicans Griseb. Cecidomyiidae Achyrocline satureioides (Lam.) DC. Cecidomyiidae Agrianthus microcilicioides Matt. Cecidomyiidae Aspilia fruticosa Baker

Cecidomyiidae Cecidomyiidae

Aspilia fruticosa Baker

Baccharis elliptica Gardner

Cecidomyiidae

Cecidomyiidae

Cecidomyiidae

Baccharis leptocephala DC. Cecidomyiidae

Cecidomyiidae

Baccharis minutiflora Mart. Ex DC. Cecidomyiidae

Lepidoptera

Baccharis platypoda DC.

Cecidomyiidae

Cecidomyiidae $\mathrm{X}$

Homoptera

NI

Cecidomyiidae

Homoptera

Baccharis pseudomyriocephala Cecidomyiidae X Teodoro

Baccharis reticularia DC.

Baccharis reticularia DC. Baccharis salzmanii DC.
Cecidomyiidae $\mathrm{X}$

Cecidomyiidae $\mathrm{X}$

Cecidomyiidae $\mathrm{X}$

Psyllidae $\mathrm{X}$

Cecidomyiidae $\mathrm{X}$

Cecidomyiidae $\mathrm{X}$

Cecidomyiidae $\mathrm{X}$

Lepidoptera $\mathrm{X}$

Cecidomyiidae $\mathrm{X}$

$\begin{array}{llll}\text { Coleoptera } & X & X & X\end{array}$

Lepidoptera $\mathrm{X} \quad \mathrm{X} \quad \mathrm{X}$

Cecidomyiidae $\mathrm{X} \quad \mathrm{X} \quad \mathrm{X}$

Cecidomyiidae $\mathrm{X} \quad \mathrm{X}$

Cecidomyiidae $\mathrm{X} \quad \mathrm{X}$

Cecidomyiidae $\mathrm{X} \quad \mathrm{X}$

Homoptera X $\mathrm{X}$

Cecidomyiidae $\mathrm{X}$

Cecidomyiidae $\mathrm{X} \quad \mathrm{X}$

Homoptera
X

$\begin{array}{lll}\text { caule } & \text { elíptica } & \text { Fig. 1D } \\ \text { folha } & \text { borda enrolada Fig. 1E }\end{array}$

folha discóide ${ }^{2}$

Fig. 1F

\begin{tabular}{|c|c|c|c|}
\hline \multirow{5}{*}{ X } & caule & elíptica & Fig. $1 \mathrm{G}$ \\
\hline & caule & elíptica & Fig. $1 \mathrm{H}$ \\
\hline & caule & globosa & Fig. 1I \\
\hline & caule & clavada & Fig. 1J \\
\hline & caule & elíptica & Fig. $1 \mathrm{~K}$ \\
\hline & caule & globosa & Fig. 1I \\
\hline $\mathrm{X}$ & folha & discóide & Fig. 1 \\
\hline$X$ & caule & elíptica & Fig. 1 \\
\hline $\mathrm{X}$ & folha & discóide & Fig. 1 \\
\hline$X$ & caule & elíptica & Fig. 11 \\
\hline & caule & elíptica & Fig. 1 \\
\hline & 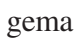 & elíptica & Fig. 1 \\
\hline
\end{tabular}

$\mathrm{X}$

folha discóide Fernandes et al. 1996

caule elíptica Fig. 1S

gema apical intumescimento Fig. 1T

folha cônica $\quad$ Fig. 2A

caule intumescimento Fig. 2B

caule globosa Fig. 2C

folha intumescimento Fig. 2D

Folha discóide Araújo et al. 2003

Caule elíptica Araújo et al. 2003

Caule globosa Araújo et al. 2003

Caule intumescimento Araújo et al. 2003

Folha legume Araújo et al. 2003

Flor intumescimento Araújo et al. 2003

Folha cônica Araújo et al. 2003

Caule globosa Araújo et al. 2003

Caule elíptica Araújo et al. 2003

Caule intumescimento Araújo et al. 2003

$\mathrm{X}$ caule elíptica Fig. $2 \mathrm{E}$

$\mathrm{X}$ caule clavada Fig. $2 \mathrm{~F}$

$\mathrm{X}$ caule globosa Fig. $2 \mathrm{G}$

folha discóide Fig. $2 \mathrm{H}$

$\mathrm{X}$ gema apical elíptica $\quad$ Fig. 2I

folha cônica $\quad$ Fig. 2J

$\mathrm{X}$ folha legume Fig. 2K

caule intumescimento Fig. 2L

caule elíptica $\quad$ Fig. $2 \mathrm{M}$

folha legume Fig. $2 \mathrm{~N}$ 
Tabela I. Continuação.

\begin{tabular}{|c|c|c|c|c|c|c|c|c|c|}
\hline Planta hospedeira & Táxon galhador & PEI & SOB & SCA & PERP PEGM & I PEBB & Órgão & Forma & Referência / Figura \\
\hline & Cecidomyiidae & $\mathrm{X}$ & & $\mathrm{X}$ & & & folha & discóide & Fig. $2 \mathrm{O}$ \\
\hline & Cecidomyiidae & & $\mathrm{X}$ & & & & caule & intumescimento & Fig. $2 \mathrm{P}$ \\
\hline \multirow[t]{4}{*}{ Baccharis serrulata (Lam.) Pers. } & Cecidomyiidae & $X$ & $\mathrm{X}$ & & & & caule & elíptica & Fig. 2Q \\
\hline & Cecidomyiidae & $\mathrm{X}$ & $\mathrm{X}$ & & & & caule & globosa & Fig. 2R \\
\hline & Cecidomyiidae & & $\mathrm{X}$ & & & & caule & globosa & Fig. 2S \\
\hline & Cecidomyiidae & & $X$ & & & & folha & globosa & Fig. 2T \\
\hline Chromolaena horminoides DC. & Cecidomyiidae & & & & & $\mathrm{X}$ & caule & elíptica & Fig. 3A \\
\hline \multirow{3}{*}{$\begin{array}{l}\text { Chromolaena multiflosculosa } \\
\text { (DC.) R. M. King \& H. Rob. }\end{array}$} & Cecidomyiidae & & & & $\mathrm{X}$ & & caule & globosa & Fig. 3B \\
\hline & Cecidomyiidae & & & & $X$ & & \multicolumn{2}{|c|}{ gema apical globosa } & Fig. 3C \\
\hline & Cecidomyiidae & & & & $X$ & & caule & elíptica & Fig. 3D \\
\hline Chromolaena sp. & Cecidomyiidae & $\mathrm{X}$ & & & & & caule & globosa & Fig. 3E \\
\hline $\begin{array}{ll}\text { Dasyphyllum } & \text { fodinarum } \\
\text { (Gardner) Cabrera } & \end{array}$ & Cecidomyiidae & & & $X$ & & & caule & elíptica & Fig. 3F \\
\hline \multirow{6}{*}{$\begin{array}{l}\text { Eremanthus erythropappus (DC.) } \\
\text { MacLeish }\end{array}$} & Cecidomyiidae & $\mathrm{X}$ & $\mathrm{X}$ & $\mathrm{X}$ & & $\mathrm{X}$ & caule & globosa & Fig. 3G \\
\hline & Tephritidae & $\mathrm{X}$ & $X$ & $X$ & & $X$ & \multicolumn{2}{|c|}{ gema apical elíptica } & Fernandes et al. 1997 \\
\hline & Tephritidae & $\mathrm{X}$ & $\mathrm{X}$ & $\mathrm{X}$ & & & caule & globosa & Fernandes et al. 1997 \\
\hline & Cecidomyiidae & $X$ & $X$ & $X$ & & $X$ & caule & elíptica & Fernandes et al. 1997 \\
\hline & Cecidomyiidae & $\mathrm{X}$ & $\mathrm{X}$ & $\mathrm{X}$ & & & gema a & lglobosa ${ }^{1}$ & Fig. 3H \\
\hline & Cecidomyiidae & $X$ & $X$ & $X$ & & $X$ & folha & globosa & Fig. 3I \\
\hline Gochnatia sp. 1 & Cecidomyiidae & & $\mathrm{X}$ & & & & caule & elíptica & Fig. 3J \\
\hline \multirow{3}{*}{$\begin{array}{l}\text { Hololepis pedunculata (DC. ex } \\
\text { Pres.) DC. }\end{array}$} & Cecidomyiidae & & & & $X$ & $X$ & folha & globosa $^{2}$ & Fig. $3 \mathrm{~K}$ \\
\hline & Tephritidae & & & & $X$ & $X$ & caule & globosa $^{2}$ & Fig. 3L \\
\hline & Cecidomyiidae & & & & $\mathrm{X}$ & & caule & intumescimento ${ }^{2}$ & Fig. 3M \\
\hline $\begin{array}{l}\text { Koanophyllon hebecladum (DC.) } \\
\text { R. M. King \& H. Rob. }\end{array}$ & Cecidomyiidae & $\mathrm{X}$ & & & & & caule & elíptica & Fig.3N \\
\hline $\begin{array}{l}\text { Lepidaploa lilacina (Mart ex DC) } \\
\text { H. Rob. }\end{array}$ & Cecidomyiidae & & & & $X$ & & caule & globosa & Fig. $3 \mathrm{O}$ \\
\hline $\begin{array}{l}\text { Lepidaploa lilacina (Mart ex DC) } \\
\text { H. Rob. }\end{array}$ & Cecidomyiidae & & & & $\mathrm{X}$ & & folha & discóide ${ }^{2}$ & Fig. 3P \\
\hline $\begin{array}{l}\text { Lessingianthus elegans (Gardner) } \\
\text { H. Rob. }\end{array}$ & Cecidomyiidae & & & & & $\mathrm{X}$ & caule & elíptica & Fig. 3Q \\
\hline $\begin{array}{l}\text { Lessingianthus linearifolius } \\
\text { (Less.) H. Rob. }\end{array}$ & Cecidomyiidae & & & $X$ & & & gema a & lglobosa & Fig. 3R \\
\hline $\begin{array}{l}\text { Lessingianthus pychnostachius } \\
\text { (DC.) H. Rob. }\end{array}$ & Cecidomyiidae & & & & $X$ & & caule & elíptica & Fig. 3S \\
\hline & Cecidomyiidae & & & & $\mathrm{X}$ & & caule & elíptica & Fig. 3T \\
\hline \multirow{3}{*}{$\begin{array}{l}\text { Lessingianthus } \\
\text { (Mart.) H. Rob }\end{array}$} & Cecidomyiidae & & $\mathrm{X}$ & & & & folha & globosa $^{1}$ & Fig. 4A \\
\hline & Cecidomyiidae & & & $\mathrm{X}$ & & & caule & globosa & Fig. 4B \\
\hline & Cecidomyiidae & & & $X$ & & & folha & discóide & Fig. 4C \\
\hline $\begin{array}{l}\text { Lessingianthus tomentellus } \\
\text { (Mart.) H. Rob }\end{array}$ & Cecidomyiidae & & & $\mathrm{X}$ & & & caule & globosa & Fig. 4D \\
\hline $\begin{array}{l}\text { Lessingianthus warmingianus } \\
\text { (Baker) H. Rob }\end{array}$ & Coleoptera & & & & $\mathrm{X}$ & & caule & globosa & Fig. 4E \\
\hline $\begin{array}{l}\text { Lourteigia ballotifolia (Kunth) R. } \\
\text { M. King \& H. Rob }\end{array}$ & Cecidomyiidae & $\mathrm{X}$ & & & & & gema a & lintumescimento & Fig. 4F \\
\hline \multirow[t]{2}{*}{ Lychnophora ericoides Mart. } & Cecidomyiidae & & $\mathrm{X}$ & & & & caule & globosa & Fig. 4G \\
\hline & Cecidomyiidae & & $\mathrm{X}$ & & & & gema a & lglobosa & Fig. $4 \mathrm{H}$ \\
\hline Lychnophora passerina Gardner & Cecidomyiidae & & & & & $\mathrm{X}$ & caule & globosa & Fig. 4I \\
\hline \multirow[t]{2}{*}{ Lychnophora ramosissima Gardner } & Cecidomyiidae & & & & $\mathrm{X}$ & & caule & elíptica & Fig. 4J \\
\hline & Cecidomyiidae & & & & $\mathrm{X}$ & & caule & globosa & Fig. 4K \\
\hline \multirow[t]{2}{*}{ Lychnophora tomentosa Sch. Bip. } & Cecidomyiidae & & & & $\mathrm{X}$ & & folha & globosa $^{1}$ & Fig. 4L \\
\hline & Cecidomyiidae & & & & $\mathrm{X}$ & & folha & legume & Fig. 4M \\
\hline \multirow{2}{*}{$\begin{array}{l}\text { Lychnophora } \\
\text { (Spreng.) Spreng, }\end{array}$} & Cecidomyiidae & $\mathrm{X}$ & & & & $\mathrm{X}$ & gema a & lelíptica ${ }^{1}$ & Fig. 4N \\
\hline & Cecidomyiidae & $X$ & & & & $X$ & caule & globosa & Fig. 40 \\
\hline
\end{tabular}


Tabela I. Continuação.

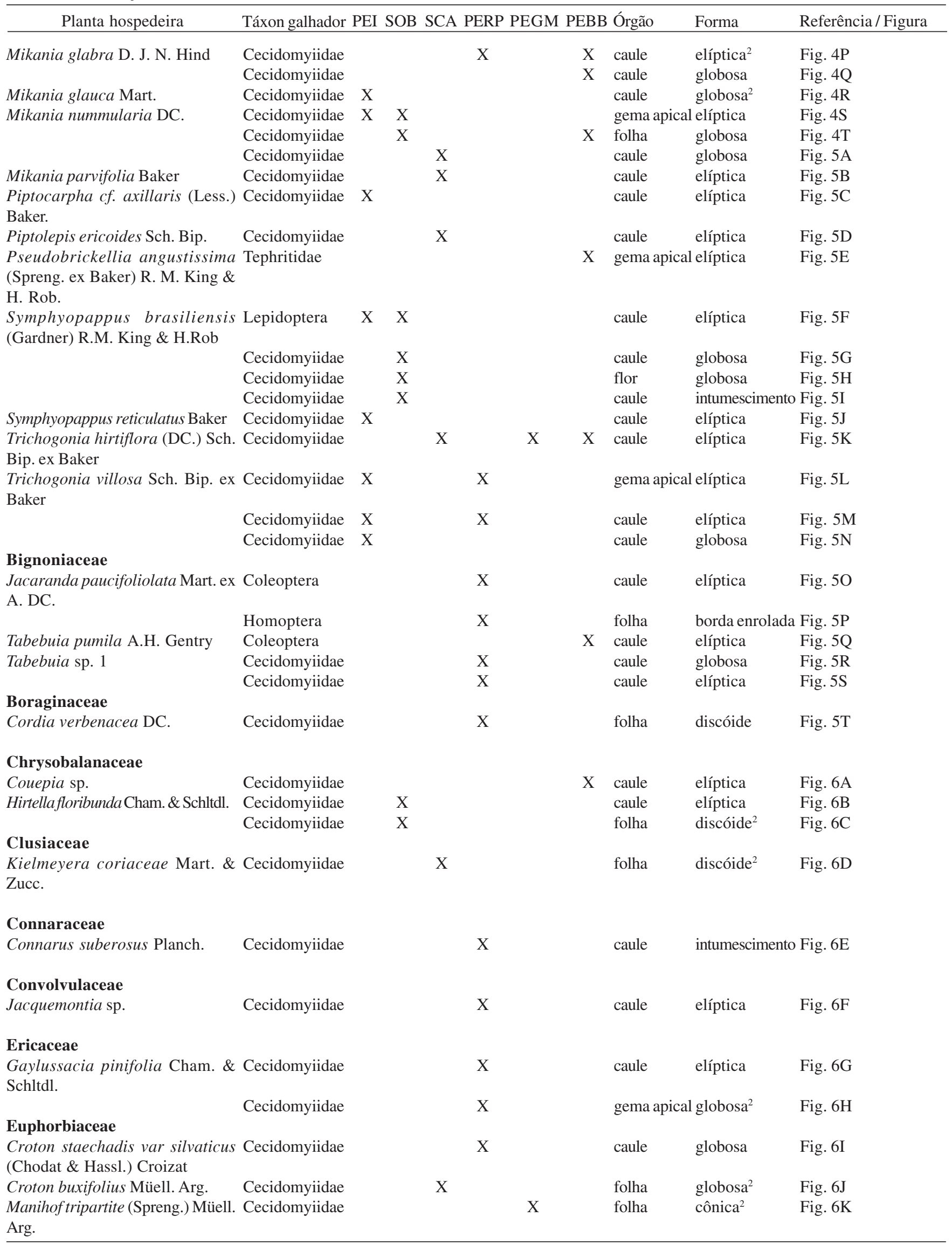


Tabela I. Continuação.

\begin{tabular}{|c|c|c|c|c|c|c|c|c|c|c|}
\hline Planta hospedeira & Táxon galhador & PEI & SOB & SCA & PERP & PEGM & PEBB & Órgão & Forma & Referência / Figura \\
\hline $\begin{array}{l}\text { Sebastiania aff. Marginata (Mart.) } \\
\text { Müell. Arg. }\end{array}$ & Cecidomyiidae & & & & $X$ & & & caule & globosa $^{2}$ & Fig. 6L \\
\hline \multicolumn{11}{|l|}{ Fabaceae } \\
\hline Bauhinia sp. 1 & Cecidomyiidae & & & & $X$ & & & caule & globosa $^{2}$ & Fig. 6M \\
\hline $\begin{array}{l}\text { Calliandra asplenioides (Ness.) R. } \\
\text { C. Barneby }\end{array}$ & Cecidomyiidae & & & & & $\mathrm{X}$ & & folha & globosa & Fig. 6N \\
\hline $\begin{array}{l}\text { Chamaecrista catharticoides (H. S. } \\
\text { Irwin \& R. C. Barneby) H. S. Irwin } \\
\text { \& R. C. Barneby }\end{array}$ & Cecidomyiidae & & & & & & $\mathrm{X}$ & caule & elíptica & Fig. 60 \\
\hline $\begin{array}{l}\text { Chamaecrista chrsysocephala (H. } \\
\text { S. Irwin \& Barneby) H. S. Irwin \& } \\
\text { Barneby }\end{array}$ & Cecidomyiidae & & & & & $\mathrm{X}$ & & caule & elíptica & Fig. 6P \\
\hline $\begin{array}{l}\text { Chamaecrista cinerascens (Benth.)H. } \\
\text { S. Irwin \& R. C. Barneby }\end{array}$ & Cecidomyiidae & & & $\mathrm{X}$ & & & & caule & elíptica & Fig. 6Q \\
\hline \multicolumn{11}{|l|}{ Fabaceae } \\
\hline $\begin{array}{l}\text { Chamaecrista cotinifolia ( G. Don) } \\
\text { H. S. Irwin \& Barneby }\end{array}$ & Cecidomyiidae & & & & $X$ & & & folha & intumescimento $^{2}$ & ${ }^{2}$ Fig. 6R \\
\hline $\begin{array}{l}\text { Chamaecrista filicifolia (Benth.) } \\
\text { H. S. Irwin \& Barneby }\end{array}$ & Cecidomyiidae & & & & $X$ & & & caule & intumescimento $^{2}$ & ${ }^{2}$ Fig. 6S \\
\hline $\begin{array}{l}\text { Chamaecrista ramosa (Vogel) H. } \\
\text { S. Irwin \& Barneby }\end{array}$ & Cecidomyiidae & & & & $\mathrm{X}$ & & & caule & intumescimento & Fig. 6T \\
\hline $\begin{array}{l}\text { Chamaecrista repens (Vogel) H. S. } \\
\text { Irwin \& R. C. Barneby }\end{array}$ & Cecidomyiidae & & & & $\mathrm{X}$ & & & caule & elíptica² & Fig. 7A \\
\hline $\begin{array}{l}\text { Chamaecrista repens (Vogel) H. S. } \\
\text { Irwin \& R. C. Barneby }\end{array}$ & Cecidomyiidae & & & & $\mathrm{X}$ & & & caule & globosa $^{2}$ & Fig. 7B \\
\hline \multirow[t]{2}{*}{ Dalbergia miscolobium Benth. } & Cecidomyiidae & & $\mathrm{X}$ & & & & & caule & elíptica & Fig. 7C \\
\hline & Cecidomyiidae & & $\mathrm{X}$ & & & & & gema apic & l globosa & Fig. 7D \\
\hline Periandra mediterranea (Vell.) & Cecidomyiidae & & $\mathrm{X}$ & $\mathrm{X}$ & & & & caule & elíptica & Fig. 7E \\
\hline Taub. & Cecidomyiidae & & $\mathrm{X}$ & $\mathrm{X}$ & & & & folha & legume & Fig. 7F \\
\hline Senna sp. & Cecidomyiidae & & & & $\mathrm{X}$ & & & caule & intumescimento & Fig. $7 \mathrm{G}$ \\
\hline \multicolumn{11}{|l|}{ Lamiaceae } \\
\hline Aegiphila lhotskiana Cham. & Cecidomyiidae & & & & & & $\mathrm{X}$ & caule & elíptica & Fig. $7 \mathrm{H}$ \\
\hline Eriope hypoleuca (Benth.) Harley & Cecidomyiidae & & & & & $\mathrm{X}$ & & caule & elíptica & Fig. 7I \\
\hline Hyptis carpinifolia Benth. & Cecidomyiidae & $\mathrm{X}$ & & & & & & caule & elíptica & Fig. 7K \\
\hline Hyptis lacunosa Pohl. ex Benth & Cecidomyiidae & & & & & & $\mathrm{X}$ & caule & elíptica & Fig. 7L \\
\hline Hyptis lippioides Pohl ex Benth. & Cecidomyiidae & & $\mathrm{X}$ & & & & & caule & intumescimento & Fig. $7 \mathrm{M}$ \\
\hline Hyptis sp1 & Cecidomyiidae & & & & $\mathrm{X}$ & & & caule & elíptica & Fig. 7N \\
\hline & Cecidomyiidae & & & & $\mathrm{X}$ & & & caule & globosa & Fig. 70 \\
\hline \multicolumn{11}{|l|}{ Lauraceae } \\
\hline Ocotea coymbosa (Meisn.) Mez & Cecidomyiidae & & & & $\mathrm{X}$ & & & folha & globosa $^{2}$ & Fig. 7P \\
\hline Ocotea glaucina (Meisn.) Mez & Cecidomyiidae & & & $\mathrm{X}$ & & & & folha & globosa ${ }^{2}$ & Fig. 7Q \\
\hline \multicolumn{11}{|l|}{ Lythraceae } \\
\hline $\begin{array}{l}\text { Cuphea ericoides Cham, \& } \\
\text { Schltdl. }\end{array}$ & Cecidomyiidae & & & & $\mathrm{X}$ & & & caule & elíptica & Fig. 7R \\
\hline \multirow[t]{2}{*}{$\begin{array}{l}\text { Diplusodon buxifolius (Cham \& } \\
\text { Schltdl.) }\end{array}$} & Cecidomyiidae & $\mathrm{X}$ & $\mathrm{X}$ & & $\mathrm{X}$ & & $\mathrm{X}$ & caule & elíptica & Fig. 7S \\
\hline & Cecidomyiidae & $X$ & $X$ & & $\mathrm{X}$ & & $\mathrm{X}$ & gema apic & l elíptica & Fig. 8A \\
\hline $\begin{array}{l}\text { Diplusodon helianthemifolius var. } \\
\text { pemphoides (DC.) Koehne }\end{array}$ & Cecidomyiidae & & & & & & $\mathrm{X}$ & caule & elíptica & Fig. 8B \\
\hline \multirow[t]{2}{*}{ Diplusodon hexander DC. } & Cecidomyiidae & & & & $\mathrm{X}$ & & $\mathrm{X}$ & terminal & elíptica & Fig. 8C \\
\hline & Cecidomyiidae & & & & $\mathrm{X}$ & & $\mathrm{X}$ & caule & elíptica & Fig. 8D \\
\hline \multirow{3}{*}{$\begin{array}{l}\text { Malpighiaceae } \\
\text { Banisteriopsis angustifolia (A. } \\
\text { Juss.) B. Gates }\end{array}$} & & & & & & & & & & \\
\hline & Cecidomyiidae & & & & & $\mathrm{X}$ & $\mathrm{X}$ & folha & discóide & Fig. 8E \\
\hline & Cecidomyiidae & & & & & & $\mathrm{X}$ & gema apic & l globosa & Fig. 8F \\
\hline
\end{tabular}


Tabela I. Continuação.

\begin{tabular}{|c|c|c|c|c|c|c|c|c|c|c|}
\hline Planta hospedeira & Táxon galhador & PEI & SOB & SCA & PERP & PEGM & PEBB & Órgão & Forma & Referência / Figura \\
\hline & Cecidomyiidae & & & & $\mathrm{X}$ & & $\mathrm{X}$ & caule & globosa & Fig. 8G \\
\hline & Thysanoptera & & & & $\mathrm{X}$ & & & folha & legume & Fig. $8 \mathrm{H}$ \\
\hline $\begin{array}{l}\text { Banisteriopsis malifolia (Nees \& } \\
\text { Mart.) B.Gates }\end{array}$ & Coleoptera & & & & & & $\mathrm{X}$ & caule & elíptica & Fig. 8I \\
\hline $\begin{array}{l}\text { Byrsonima arctostaphylloides } \\
\text { Nied. }\end{array}$ & Coleoptera & & & & $\mathrm{X}$ & & & caule & elíptica & Fig. 8J \\
\hline Byrsonima clausseniana A. Juss. & Cecidomyiidae & & & & $\mathrm{X}$ & & & caule & globosa & Fig. 8K \\
\hline \multirow[t]{8}{*}{ Byrsonima coccolobifolia Kunth } & Cecidomyiidae & & & $X$ & & $\mathrm{X}$ & $\mathrm{X}$ & caule & elíptica & Fig. 8L \\
\hline & Hymenoptera & $\mathrm{X}$ & & $\mathrm{X}$ & $\mathrm{X}$ & $\mathrm{X}$ & $\mathrm{X}$ & caule & globosa & $\begin{array}{l}\text { Gonçalves-Alvim \& } \\
\text { Fernandes } 2001 \mathrm{~b}\end{array}$ \\
\hline & Cecidomyiidae & & & $\mathrm{X}$ & & $\mathrm{X}$ & $\mathrm{X}$ & folha & discóide & Fig. $8 \mathrm{M}$ \\
\hline & Cecidomyiidae & $\mathrm{X}$ & & $X$ & & $\mathrm{X}$ & $\mathrm{X}$ & folha & elíptica & Fig. $8 \mathrm{~N}$ \\
\hline & Cecidomyiidae & & & $\mathrm{X}$ & & $\mathrm{X}$ & & gema apical & intumescimento & Fig. 80 \\
\hline & Cecidomyiidae & $\mathrm{X}$ & & & $\mathrm{X}$ & $\mathrm{X}$ & $\mathrm{X}$ & caule & intumescimento & Fig. 8P \\
\hline & Cecidomyiidae & & & $\mathrm{X}$ & $\mathrm{X}$ & $\mathrm{X}$ & $X$ & folha & cônica ${ }^{2}$ & Fig. 8Q \\
\hline & Homoptera & & & $X$ & & & & folha & legume & Fig. 8R \\
\hline \multirow[t]{2}{*}{ Byrsonima variabilis A. Juss. } & Cecidomyiidae & $\mathrm{X}$ & & $\mathrm{X}$ & & & & caule & globosa & Fig. 8S \\
\hline & Cecidomyiidae & & & $\mathrm{X}$ & & & & caule & intumescimento & Fig. 8T \\
\hline Byrsonima variabilis A. Juss. & Cecidomyiidae & $\mathrm{X}$ & & & & & & caule & globosa $^{2}$ & Fig. 9A \\
\hline \multirow[t]{3}{*}{ Byrsonima sp. 1} & Cecidomyiidae & & & $\mathrm{X}$ & & & & caule & elíptica & Fig. 9B \\
\hline & Cecidomyiidae & & & $\mathrm{X}$ & & & & folha & globosa ${ }^{2}$ & Fig. 9C \\
\hline & Cecidomyiidae & & & $\mathrm{X}$ & & & & caule & globosa & Fig. 9D \\
\hline $\begin{array}{l}\text { Heteropteris byrsonimiifolia A. } \\
\text { Juss }\end{array}$ & Cecidomyiidae & & $\mathrm{X}$ & & & & & folha & discóide & $\begin{array}{l}\text { Fernandes et } \\
\text { al.1997 }\end{array}$ \\
\hline Peixotoa cipoana C. E. Anderson & Cecidomyiidae & & & & & & $\mathrm{X}$ & folha & globosa & Fig. 9E \\
\hline \multicolumn{11}{|l|}{ Melastomataceae } \\
\hline Leandra aurea (Cham.) Cogn. & Tephritidae & $\mathrm{X}$ & & & & & & caule & globosa & Fig. 9F \\
\hline \multirow{2}{*}{ Macairea radula DC. } & Lepidoptera & $\mathrm{X}$ & & $\mathrm{X}$ & & & $\mathrm{X}$ & folha & globosa ${ }^{1}$ & Fig. 9G \\
\hline & Lepidoptera & & $\mathrm{X}$ & $\mathrm{X}$ & & & & caule & elíptica ${ }^{1}$ & Fig. 9H \\
\hline \multirow[t]{3}{*}{ Marcetia taxifolia (A. St.-Hil.) DC. } & Cecidomyiidae & & & $\mathrm{X}$ & & & $\mathrm{X}$ & caule & elíptica & Fig. 9I \\
\hline & Cecidomyiidae & & & $X$ & $\mathrm{X}$ & & $X$ & gema apical & elíptica ${ }^{1}$ & Fig. 9J \\
\hline & Cecidomyiidae & & & & & & $\mathrm{X}$ & caule & globosa & Fig. 9K \\
\hline Marcetia hirsuta Cogn. & Cecidomyiidae & & $\mathrm{X}$ & & & & & gema apical & elíptica ${ }^{1}$ & Fig. 9L \\
\hline Miconia albicans (Sw.) Steud & Nematoda & & $\mathrm{X}$ & & & & & folha & globosa & Fig. 9M \\
\hline Miconia corallina Spring & Nematoda & & & $\mathrm{X}$ & & & & folha & globosa $^{2}$ & Fig. 9N \\
\hline \multirow[t]{3}{*}{ Miconia sp. 1} & Cecidomyiidae & & $\mathrm{X}$ & & & & & folha & discóide ${ }^{2}$ & Fig. 90 \\
\hline & Cecidomyiidae & $\mathrm{X}$ & & & & & & caule & elíptica & Fig. 9P \\
\hline & Cecidomyiidae & $\mathrm{X}$ & & & & & & caule & globosa & Fig. 9Q \\
\hline Miconia sp. 2 & Cecidomyiidae & $\mathrm{X}$ & & & & & & caule & intumescimento & Fig. 9R \\
\hline Microlicia cardiophora Naud. & Cecidomyiidae & & & & & & $\mathrm{X}$ & caule & elíptica & Fig. 9S \\
\hline \multirow[t]{2}{*}{ Microlicia confertiflora Naud. } & Cecidomyiidae & & & & & & $\mathrm{X}$ & caule & elíptica & Fig. 9T \\
\hline & Cecidomyiidae & & & & & & $\mathrm{X}$ & caule & globosa & Fig. 10A \\
\hline Microlicia euphorbioides Mart. & Cecidomyiidae & & & & $\mathrm{X}$ & & & caule & elíptica & Fig. 10B \\
\hline Microlicia fasciculata Mart. & Lepidoptera & & & & & $\mathrm{X}$ & & caule & clavada & Fig. 10C \\
\hline Microlicia graveolens DC. & Cecidomyiidae & & & & & $\mathrm{X}$ & & gema apical & elíptica ${ }^{1}$ & Fig. 10D \\
\hline Microlicia multicaules Mart. & Cecidomyiidae & & & $\mathrm{X}$ & & & & caule & elíptica & Fig. 10E \\
\hline $\begin{array}{l}\text { Microlicia suborbicularifolia } \\
\text { Hoehne }\end{array}$ & Cecidomyiidae & & & $\mathrm{X}$ & & & & caule & elíptica & Fig. 10F \\
\hline Microlicia sp. 1 & Cecidomyiidae & & & & & & $\mathrm{X}$ & caule & elíptica & Fig. 10G \\
\hline Microlicia sp. 2 & Cecidomyiidae & & & & $\mathrm{X}$ & & & caule & globosa & Fig. $10 \mathrm{H}$ \\
\hline Tibouchina arenaria Cogn. & Cecidomyiidae & & $\mathrm{X}$ & & & & & caule & intumescimento & Fig. 10I \\
\hline Tibouchina multiflora Cogn. & Cecidomyiidae & & $X$ & & & & & caule & elíptica & Fig. 10J \\
\hline Trembleya laniflora Cogn. & Cecidomyiidae & & & $\mathrm{X}$ & & & & gema apical & globosa $^{1}$ & Fig. $10 \mathrm{~K}$ \\
\hline $\begin{array}{l}\text { Trembleya parviflora (Don) } \\
\text { Cogn. }\end{array}$ & Cecidomyiidae & $\mathrm{X}$ & & & & & & caule & globosa & Fig. 10L \\
\hline
\end{tabular}


Tabela I. Continuação.

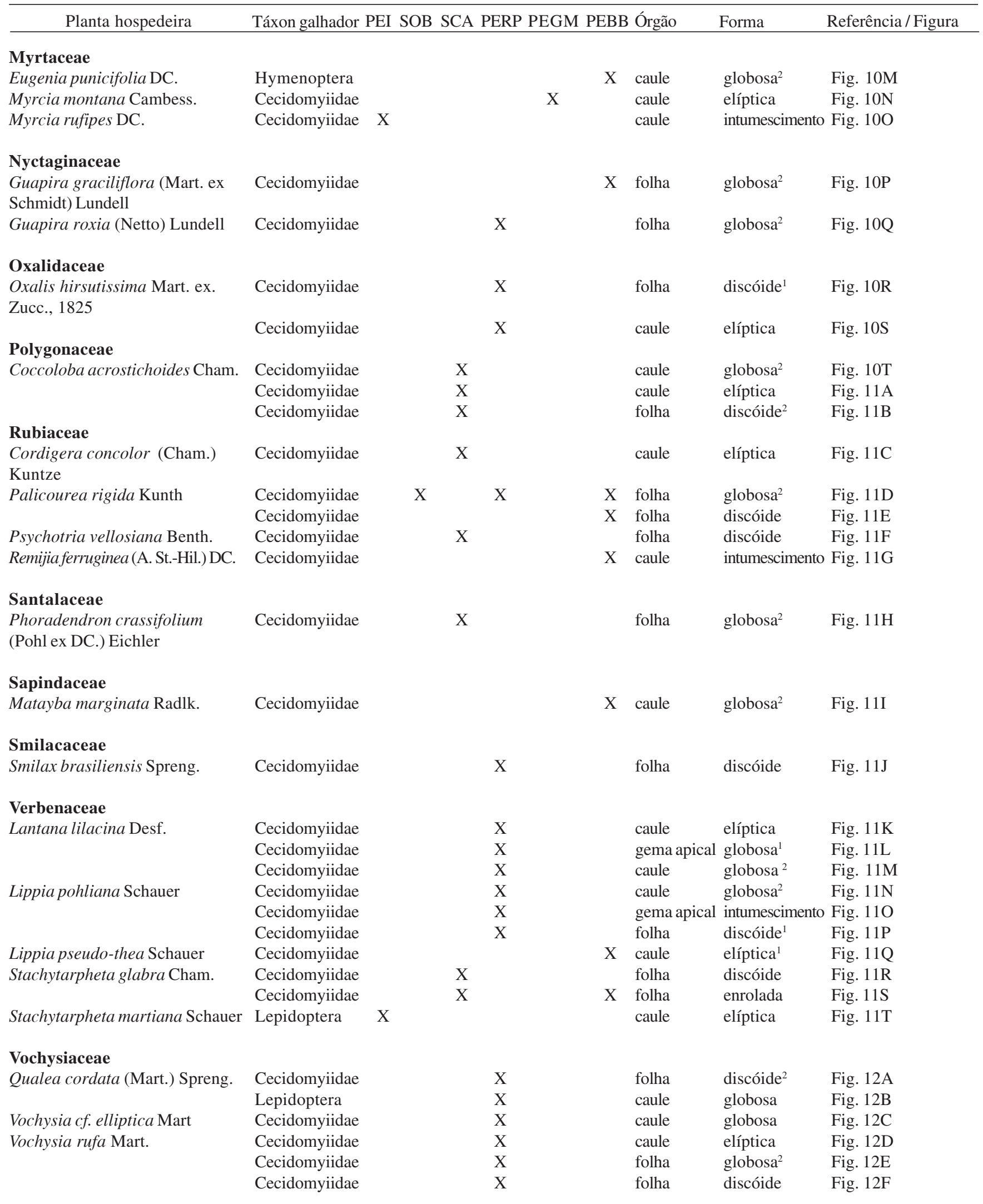

${ }^{1}$ galha pubescente, ${ }^{2}$ galha de ocorrência agrupada. 

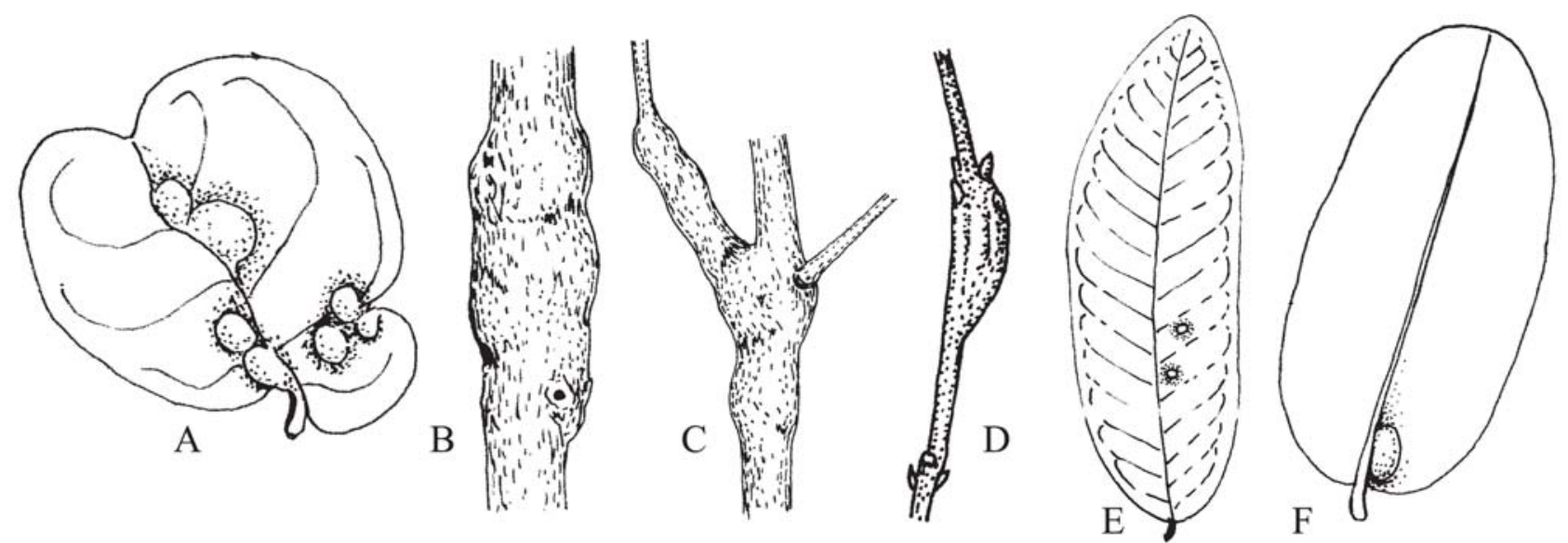

Fig. 12. Morfotipos de galhas em campos rupestres na porção sul da Cadeia do Espinhaço. Para identificação das plantas hospedeiras veja a Tabela I. Vochysiaceae: Qualea cordata (A-B), Vochysia cf. eliptica (C), V. rufa (D-F).

Tabela II. Número de morfotipos de galhas associados às famílias de plantas em campos rupestres da Cadeia do Espinhaço, MG. As famílias que não apresentaram nenhuma galha foram indicadas como 'outras famílias' (Erythroxylaceae, Gesneriaceae, Meliaceae, Velloziaceae, Annonaceae, Flacourtiaceae, Dilleniaceae, Eriocaulaceae, Malvaceae, Moraceae, Myrsinaceae, Ochinaceae, Proteaceae, Rutaceae, Campanulaceae, Caryocaraceae, Gentianaceae, Krameriaceae, Orchidaceae, Salicaceae, Scrophulariaceae, Solanaceae, Sterculiaceae, Winteraceae).

\begin{tabular}{|c|c|c|c|c|}
\hline \multirow[t]{2}{*}{ Famílias } & \multicolumn{2}{|c|}{ Plantas hospedeiras } & \multicolumn{2}{|c|}{ Galhas } \\
\hline & riqueza & $\%$ & riqueza & $\%$ \\
\hline Asteraceae & 95 & 24,74 & 102 & 42,32 \\
\hline Melastomataceae & 42 & 10,9 & 27 & 11,20 \\
\hline Fabaceae & 26 & 6,77 & 15 & 6,22 \\
\hline Rubiaceae & 22 & 5,73 & 5 & 2,07 \\
\hline Malpighiaceae & 18 & 4,69 & 23 & 9,54 \\
\hline Euphorbiaceae & 15 & 3,91 & 4 & 1,66 \\
\hline Lamiaceae & 13 & 3,39 & 8 & 3,32 \\
\hline Myrtaceae & 12 & 3,13 & 3 & 1,24 \\
\hline Lythraceae & 9 & 2,34 & 6 & 2,49 \\
\hline Apocynaceae & 9 & 2,34 & 2 & 0,83 \\
\hline Ericaceae & 8 & 2,08 & 2 & 0,83 \\
\hline Verbenaceae & 7 & 1,82 & 10 & 4,15 \\
\hline Aquifoliaceae & 6 & 1,56 & 2 & 0,83 \\
\hline Clusiaceae & 6 & 1,56 & 1 & 0,41 \\
\hline Vochysiaceae & 5 & 1,30 & 6 & 2,49 \\
\hline Chrysobalanaceae & 5 & 1,30 & 3 & 1,24 \\
\hline Polygonaceae & 4 & 1,04 & 3 & 1,24 \\
\hline Nyctaginaceae & 4 & 1,04 & 2 & 0,83 \\
\hline Bignoniaceae & 3 & 0,78 & 5 & 2,07 \\
\hline Lauraceae & 3 & 0,78 & 2 & 0,83 \\
\hline Connaraceae & 2 & 0,52 & 1 & 0,41 \\
\hline Sapindaceae & 2 & 0,52 & 1 & 0,41 \\
\hline Oxalidaceae & 1 & 0,26 & 2 & 0,83 \\
\hline Anacardiaceae & 1 & 0,26 & 1 & 0,41 \\
\hline Araliaceae & 1 & 0,26 & 1 & 0,41 \\
\hline Boraginaceae & 1 & 0,26 & 1 & 0,41 \\
\hline Convolvulaceae & 1 & 0,26 & 1 & 0,41 \\
\hline Santalaceae & 1 & 0,26 & 1 & 0,41 \\
\hline Smilacaceae & 1 & 0,26 & 1 & 0,41 \\
\hline Outras famílias & 48 & 12,50 & 0 & 0 \\
\hline Não determinado & 13 & 3,39 & 0 & 0 \\
\hline Total & 384 & 100 & 241 & 100 \\
\hline
\end{tabular}

para oviposição por não serem sujeitos ao dano mecânico causado por vento ou por outros herbívoros. Uma outra possibilidade para explicar o número maior de espécies de insetos indutores de galhas em ramos além da proteção seria a qualidade nutricional dos tecidos da galha. Os padrões filogenéticos de afídeos e vespas sugerem que a radiação adaptativa destes grupos seguiu a tendência de centralização dos sítios de oviposição em resposta a qualidade nutricional da planta (Inbar et al. 2004; Price 2005). Ramos, pecíolos e gemas são mais centrais do que folhas em relação ao sistema vascular da planta. As larvas manipulam mais facilmente a fisiologia da planta drenando mais nutrientes dos tecidos sadios da planta para a galha. Assim, as larvas conseguem um alimento de melhor qualidade nutricional, e consequentemente, maior sucesso reprodutivo (Price 2005). Em afídeos, por exemplo, galhas primitivas formadas em folhas sustentam poucos indivíduos, enquanto que as galhas induzidas em gemas apicais sustentam milhares de indivíduos (Inbar et al. 2004). Entretanto, esta hipótese ainda não foi testada e estudos observacionais e experimentais devem ser realizados para elucidar esta questão.

Este é o primeiro estudo feito em diferentes serras da região Neotropical utilizando uma amostragem padronizada. Outras serras deverão ser amostradas, para que seja alcançada uma melhor compreensão da distribuição dos insetos galhadores e de suas plantas hospedeiras ao longo da Cadeia do Espinhaço. Mesmo sendo estes resultados parciais, devido à grande extensão territorial dos campos rupestres, e pelo fato de que este trabalho considerar apenas as espécies de insetos indutores de galhas em plantas lenhosas menores que $2 \mathrm{~m}$ de altura, pode-se dizer que a riqueza dos insetos indutores de galhas corrobora a afirmação de que campo rupestre é um dos hábitats com maior diversidade de insetos galhadores ao lado de outras regiões como os "fynbos" na África do Sul.

Agradecimentos. Agradecemos a M. S. Coelho, D. Negreiros, F. A. O. Silveira e a dois revisores anônimos pela revisão crítica e sugestões ao manuscrito. Ao Instituto Estadual de Florestas I.E.F. pelo suporte logístico. A FAPEMIG (2893/98) e ao CNPq (472811/2006-1; 30.9633/ 
2007-9) pelo suporte financeiro. A CAPES/PICDT pela bolsa de estudos concedida a M. A. A. Carneiro. Este trabalho foi parte dos requerimentos para conclusão do curso de Doutorado em Ecologia, Conservação e Manejo da Vida Silvestre por M. A. A. Carneiro, na Universidade Federal de Minas Gerais.

\section{REFERÊNCIAS}

Abrahamson, W. G. \& A. E. Weis. 1997. Evolutionary ecology across three trophic levels: goldenrods, gallmakers and natural enemies. New Jersey, Princeton University Press, xvi+456 p.

Almeida, A. M.; P. I. Prado \& T. M. Lewinsohn. 2004. Geographical distribution of Eupatorieae (Asteraceae) in south-eastern and south Brazilian mountain ranges. Plant Ecology 174: 163-181.

APG II. 2003. An update of the Angiosperm phylogeny group classification for the orders and families of flowering plants: APGII. Botanical Journal of the Linnean Society 141: 399-436.

Araújo, A. P. A.; M. A. A. Carneiro \& G. W. Fernandes. 2003. Efeitos do sexo, do vigor e do tamanho da planta hospedeira sobre a distribuição de insetos indutores de galhas em Baccharis pseudomyriocephala Teodoro (Asteraceae). Revista Brasileira de Entomologia 47: 483-490.

Barroso, G. M. 1976. Compositae - Subtribo Baccharidinae Hoffman Estudo das espécies ocorrentes no Brasil. Rodriguésia 27: 3273.

Benites, V. M.; C. E. Schaefer; F. N. B. Simas \& H. G. Santos. 2007. Soils associated with rock outcrops in the Brazilian mountain ranges. Revista Brasileira de Botânica 30: 569-577.

Carneiro M. A. A.; C. S. A. Branco; C. E. D. Braga; E. D. Almada; M. B. M. Costa; V. C. Maia \& G. W. Fernandes. 2009. Are gall midge species (Diptera, Cecidomyiidae) host plant-specialists? Revista Brasileira de Entomologia 53: 365-378.

Coelho M. S.; E. D. Almada; G. W. Fernandes; M. A. A. Carneiro; R. M. Santos; A. V. Quintino \& A. Sanchez-Azofeifa. 2009. Gall inducing arthropods from a seasonally dry tropical forest in Serra do Cipó, Brazil. Revista Brasileira de Entomologia 53: 404-414.

Cuevas-Reyes, P.; M. Quesada; P. Hanson; R. Dirzo \& K. Oyama. 2004. Diversity of gall-inducing insects in a Mexican tropical dry Forest: the importance of plant species richness, life forms, host plant age and plant density. Journal of Ecology 92: 707-716.

Derby, D. A. 1906. The Serra do Espinhaço, Brazil. Journal of Geology 14: $374-401$.

Dreger-Jauffret, F. \& J. D. Shorthouse. 1992. Diversity of gall-inducing insects and their galls, p. 8-33. In: J. D. Shorthouse \& O. Rohfritsch (eds.). Biology of Insect-Induced Galls, New York, Oxford University Press, $x i+285 \mathrm{p}$.

Espírito-Santo, M. M. \& G. W. Fernandes. 2007. How many species of gall-inducing insects are there on earth, and where are there? Annals of the Entomological Society of America 100: 95-99.

Fernandes G. W. 1992. Plant family size and age effects on insular gallforming species richness. Global Ecology and Biogeography letters 2: 71-74.

Fernandes, G. W.; M. A. A. Carneiro; A. C. F. Lara; L. R. Allain; G. R. Julião; G. I. Andrade; T. R. Reis \& I. M. Silva. 1996. Galling insects on neotropical species of Baccharis (Asteraceae). Tropical Zoology 9: 315-332.

Fernandes, G. W.; R. C. Araújo; S. C. Araújo; J. A. Lombardi; A. S. Paula; R. Loyola Júnior \& T. G. Cornelissen. 1997. Insect galls from savanna and rocky fields of the Jequitinhonha valley, Minas Gerais, Brazil. Naturalia 22: 221-244.

Fernandes, G. W.; G. R. Julião; R. C. Araújo; S. C. Araújo; J. A. Lombardi; D. Negreiros \& M. A. A. Carneiro. 2001. Distribution and morphology of insect galls of the Rio Doce Valley, Brazil. Naturalia 26: 211-244

Fernandes, G. W. \& P. W. Price. 1988. Biogeographical gradients in galling species richness: tests of hypotheses. Oecologia 76: 161167.

Floate, K. D.; G. W. Fernandes \& J. A. Nilsson. 1996. Distinguishing intrapopulacional categories of plants by their insect faunas: galls on rabbitbrush. Oecologia 105: 221-229.

Gagné, R. J. 1994. The gall midges of the region neotropical. Ithaca, Comstock, xiv+352 p.

Galvão, M. V. \& E. Nimer. 1965. Clima, p. 91-139. In: IBGE (ed.). Geografia do Brasil - Grande região leste. Rio de janeiro, IBGE, vii+486 p.

Giulietti, A. M; N. L. Menezes; J. R. Pirani; M. L. Meguro \& M. G. L. Wanderley. 1987. Flora da Serra do Cipó: Caracterização e lista de espécies. Boletim Botânica Universidade São Paulo 9: 1152.

Giulietti, A. M. \& J. R. Pirani. 1988. Patterns of Geographic Distribution of some Plant Species from the Espinhaço Range, Minas Gerais, Brazil, p. 39-69. In: P. E. Vanzolini \& W. R. Heyer (eds.). Proceedings of a Workshop on Neotropical Biodiversity Distribution Patterns. Rio de Janeiro, Academia Brasileira de Ciências, iv+488 p.

Gonçalves-Alvim, S. J. \& G. W. Fernandes. 2001a. Biodiversity of galling insects: historical, community and habitat effects in four neotropical savannas. Biodiversity and Conservation. 10: 7998.

Gonçalves-Alvim, S. J. \& G. W. Fernandes. 2001b. Comunidades de insetos galhadores (Insecta) em diferentes fisionomias do cerrado em Minas Gerais, Brasil. Revista Brasileira Zoologia 18: 289305.

Hatschbach, G.; E. A. E. Guarçoni; M. A. Sartori \& O. S. Ribas. 2006. Aspectos fisionômicos da vegetação da Serra do Cabral - Minas Gerais - Brasil. Boletim do Museu Botânico Municipal de Curitiba 67: 1-33.

Hawkins, B. A. \& S. G. Compton. 1992. African fig wasp communities: undersaturation and latitudinal gradients in species richness. Journal of Animal Ecology 61: 361-372.

Hind, D. J. N. 2003. Flora of Grão-Mogol, Minas Gerais: Compositae (Asteraceae). Boletim Botânica Universidade São Paulo 21: $179-234$

Holmgren P. K.; N. H. Holmgren \& L. C. Barnett. 1990. Index Herbariorum. Part I: The Herbaria of The World. New York, New York Botanical Garden, vi+693 p.

Inbar, M.; M. Wink \& D. Wool. 2004. The evolution of host plant manipulation by insects: molecular and ecological evidence from gall-forming aphids on Pistacia. Molecular Phylogenetics and Evolution 32: 504-511.

Jepson W. 2005. A disappearing biome? Reconsidering land-cover change in the Brazilian savanna. The Geographical Journal 171: 99111 .

Julião, G. R.; M. E. C. Amaral \& G. W. Fernandes. 2002. Galhas de insetos e suas plantas hospedeiras no Pantanal sul-mato-grossense. Naturalia 27: 47-74.

Julião, G. R.; E. M. Venticinque; G. W. Fernandes \& J. E. Kraus. 2005. Richness and abundance of gall-forming insects in the Mamirauá Varzea, a flooded Amazoniam forest. Uakari 1: 39-42.

Lara, A. C. F. \& G. W. Fernandes. 1996. The highest diversity of galling insects: Serra do Cipó, Brazil. Biodiversity Letters 3: 111-114

Maia, V. C. 2005. Catálogo dos Cecidomyiidae (Diptera) do Estado do Rio de Janeiro. Biota Neotropica 5: 1-15.

Maia, V. C. \& G. W. Fernandes. 2004. Insect galls from Serra de São José (Tiradentes, Minas Gerais, Brazil). Brazilian Journal Biology 64: 423-445.

Mendonça, M. S. 2007. Plant diversity and galling arthropod diversity searching for taxonomic patterns in an animal-plant interaction in the neotropics. Boletín Sociedad Argentina Botánica 42: $347-357$.

Moreira, A. N. 1977. Relevo, p. 1-45. In: IBGE (ed.). Geografia do Brasil. Região Nordeste. Rio de Janeiro, IBGE, iii+105 p.

Munhoz, C. B. R. \& C. E. B. Proença. 1998. Composição florística do município de Alto Paraíso de Goiás na Chapada dos Veadeiros. Boletim Herbário Ezechias Paulo Heringer 3: 102-150.

Myers, N.; C. G. Mittermeier; G. A. B. Fonseca \& J. Kent. 2000. Biodiversity hotspots for conservation priorites. Nature 403: 853858 . 
Nyman, T. \& R. Julkunen-Tiitto. 2000. Manipulation of the phenolic chemistry of willows by gall-inducing sawflies. Proceedings National Academic of Science USA 97: 13184-13187.

Oliveira, P. S. \& R. J. Marquis. 2002. The Cerrados of Brazil: ecology and Natural history of a Neotropical savanna. New York, Columbia University Press, $\mathrm{x}+398 \mathrm{p}$.

Oliveira-Filho, A. \& J. A. Ratter. 2002. Vegetation physiognomies and woody flora of the Cerrado Biome, p. 91-120. In: P. S. Oliveira \& R. J. Marquis (eds.). The Cerrados of Brazil: ecology and Natural history of a Neotropical savanna. New York, Columbia University Press, $\mathrm{x}+398 \mathrm{p}$.

Pirani, J. R.; R. Mello-Silva \& A. M. Giulietti. 2003. Flora de GrãoMogol, Minas Gerais, Brasil. Boletim de Botânica da Universidade de São Paulo 21: 1-24.

Price, P. W. 2005. Adaptative radiation of gall-inducing insects. Basic and Applied Ecology 6: 413-421.

Price, P. W.; G. W. Fernandes; A. C. F. Lara; J. Brawn; D. Gerling; H. Barrios; M. G. Wright; S. P. Ribeiro \& N. Rothcliff. 1998. Global patterns in local number of insect galling species. Journal of Biogeography 25: 581-591.

Price, P. W.; H. Roininen \& A. Zinovjev. 1997. Adaptative radiation of gall-inducing sawflies in relation to architecture and geographic range of willow host plants, p. 196-203. In: G. Csóka; W. J. Mattson; G. N. Stone \& P. W. Price (eds.). Biology of gall-inducing arthropods. St. Paul, USDA Tech., Rep. NC-199.

Price, P. W.; G. L. Waring \& G. W. Fernandes. 1986. Hypotheses on the adaptive nature of galls. Proceedings of the Entomological Society of Washington 88: 361-363.

Raman, A. 2007. Insect-induced plant galls of India: unresolved questions. Current Science 92: 748-757.

Rapini, A.; R. Mello-Silva \& M. L. Kawasaki. 2002. Richness and endemism in Asclepiadoideae (Apocynaceae) from the Espinhaço Range of Minas Gerais, Brazil - a conservationist view. Biodiversity and Conservation 11: 1733-1746.

Ratter, J. A.; S. Bridgewater \& J. F. Ribeiro. 2006. Biodiversity patterns of the woody vegetation of the Brazilian Cerrados, p. 31-66. In: R. T. Pennington; G. P. Lewis \& J. A. Ratter (eds.). Neotropical Savannas and seasonally dry forests: plant diversity, biogeography, and conservation. Boca Raton, The Systematics Association Special Volume Series 69. CRC Press, $x+484$ p.
Ronquist, F. 1994. Evolution of parasitism among closely related species: phylogenetic relationships and the origin of inquilinism in gall wasps (Hymenoptera, Cynipidae). Evolution 48: 241-266.

Safford, H. D. 1999. Brazilian Páramos I. An introduction to the physical environment and vegetation of the campos de altitude. Journal of Biogeography 26: 693-712.

Shorthouse, J. D.; D. Wool \& A. Raman. 2005. Gall-inducing insects Nature's most sophisticated herbivores. Basic and Applied Ecology 6: 407-411.

Silva, J. F.; M. R. Farinas; J. M. Felfili \& C. A. Klink. 2006. Spatial heterogeneity, land use and conservation in the cerrado region of Brazil. Journal of Biogeography 33: 536-548.

Stone, G. N. \& K. Schönrogge. 2003. The adaptive significance of insect gall morphology. Trends Ecology and Evolution 18: 512 522.

Stone, G. N.; K. Schönrogge; R. J. Atkinson; D. Bellido \& J. PujadeVillar. 2002. The population biology of oak gall wasps (Hymenoptera: Cynipidae). Annual review Entomology 47: 633668.

Urso-Guimarães, M. V. \& C. Scarelli-Santos. 2006. Galls and gall makers in plants from the Pé-de-Gigante Cerrado Reserve, Santa Rita do Passa Quatro, SP, Brazil. Brazilian Journal of Biology 66 357-369.

Urso-Guimarães, M. V.; C. Scareli-Santos \& A. C. Bonifácio-Silva. 2003. Occurrence and characterization of entomogen galls in plants from natural vegetation areas in Delfinópolis, MG, Brazil. Brazilian Journal of Biology 63: 705-715

Veldtman, R. \& M. A. McGeoch. 2003. Gall-forming insect species richness along a non-scleromorphic vegetation rainfall gradient in South Africa: the importance of plant community composition. Austral Ecology 28: 1-13.

Zappi D. C.; E. Lucas; B. L. Stannard; E. N. Lughadha; J. R. Pirani; L. P. Queiroz; S. Atkins; D. J. N. Hind; A. M. Giulietti; R. M. Harley \& A. M. Carvalho. 2003. Lista das Plantas Vasculares de Catolés, Chapada Diamantina, Bahia, Brasil. Boletim Botânica Universidade São Paulo 21: 345-398.

Weis, A. E.; R. Walton \& C. L. Crego. 1988. Reactive plant tissue sites and the population biology of gall makers. Annual Review of Entomology 33: 467-486.

Recebido em 27/08/2008; aceito em 12/06/2009 